\title{
The lubrication approximation for thin viscous films: the moving contact line with a 'porous media' cut-off of van der Waals interactions
}

\author{
A L Bertozzi $†$ and $M$ Pugh $\ddagger \|$ \\ $\dagger$ Department of Mathematics, The University of Chicago, Chicago, minois 60637, USA \\ $\ddagger$ Courant Institute, New York University, New York, NY 10012, USA
}

Received 10 June 1994

Recommended by P Constantin

\begin{abstract}
We consider the effect of a second-order 'porous media' term on the evolution of weak solutions of the fourth-order degenerate diffusion equation$$
h_{t}=-\nabla \cdot\left(h^{n} \nabla \Delta h-\nabla h^{m}\right)
$$

in one space dimension. The equation without the second-order term is derived from a 'lubrication approximation' and models surface tension dominated motion of thin viscous films and spreading droplets. Here $h(x, t)$ is the thickness of the film, and the physical problem corresponds to $n=3$.

For simplicity, we consider periodic boundary conditions which has the physical interpretation of modelling a periodic array of droplets. In a previous work we studied the above equation without the second-order 'porous media' term. In particular we showed the existence of non-negative weak solutions with increasing support for $0<n<3$ but the techniques failed for $n \geqslant 3$. This is consistent with the fact that, in this case, non-negative self-similar source-type solutions do not exist for $n \geqslant 3$.

In this work, we discuss a physical justification for the 'porous media' term when $n=3$ and $1<m<2$. We propose such behaviour as a cut off of the singular 'disjoining pressure' modelling long range van der Waals interactions.

For all $n>0$ and $1<m<2$, we discuss possible behaviour at the edge of the support of the solution via leading order asymptotic analysis of travelling wave solutions. This analysis predicts a certain 'competition' between the second-and fourth-order terms. We present rigorous weak existence theory for the above equation for all $n>0$ and $1<m<2$. In particular, the presence of a second-order 'porous media' term in the above equation yields non-negative weak solutions that converge to their mean as $t \rightarrow \infty$ and that have additional regularity. Moreover, we show that there exists a time $T^{*}$ after which the weak solution is a positive strong solution. For $n>3 / 2$, we show that the regularity of the weak solutions is in exact agreement with that predicted by the asymptotics.

Finally, we present several numerical computations of solutions. The simulations use a weighted implicit-explicit scheme on a dynamically adaptive mesh. The numerics suggest that the weak solution described by our existence theory has compact support with a finite speed of propagation. The data confirms the local 'power law' behaviour at the edge of the support predicted by asymptotics.
\end{abstract}

AMS classification scheme numbers: $35 \mathrm{~K} 55$, 35L 75

PACS numbers: $6845 \mathrm{G}, 0340 \mathrm{G}, 4710,6810 \mathrm{G}, 6810 \mathrm{C}$

$\S$ E-mail:bertozzi@math.uchicago.edu

|| E-mail:pugh@aloss.cims.nyu.edu 


\section{Introduction}

We consider weak solutions of the fourth-order degenerate diffusion equation

$$
h_{t}=-\nabla \cdot\left(f(h) \nabla \Delta h-\nabla h^{m}\right)
$$

in one space dimension. We study the case where $f(h)=h^{n}, n>0$. This equation is relevant to surface tension dominated motion of thin viscous films and spreading droplets [18]. The second-order term in the equation, $\Delta h^{m}$, arises as a cut off of van der Waals interactions. We refer to it as the 'porous media' term since it is the nonlinearity that arises in the well known 'porous media' equation [25]. Physically, $h$ is the thickness of the the film. This paper considers weak solutions that can be zero on a set of non-zero measure, hence are relevant to the droplet problem. We show that for $n \geqslant 3$, the inclusion of such a 'porous media' term removes the singularity associated with the movement of the 'contact line' identified in a previous work [6].

\subsection{Long range van der Waals interactions and the lubrication approximation}

The lubrication approximation for a thin film of liquid on a solid surface yields a fourthorder degenerate diffusion equation for the film height [18]. In one space dimension, with a no slip boundary condition on the liquid solid surface, it is

$$
h_{t}+\left(|h|^{3} h_{x x x}\right)_{x}=0 \text {. }
$$

The derivation uses the Stokes equation for steady viscous flow combined with a depth averaging of the fluid velocity in the direction perpendicular to the surface.

The no slip boundary condition causes a paradox for films with a moving edge or contact line. Indeed, a moving contact line produces infinite energy dissipation [17, 19]. Many authors have considered ways of addressing this problem, including the use of a 'slip condition' on the liquid/solid interface. In [6], we discussed the 'slip models' which mathematically add a term of the form $\beta|h|^{p}, 0<p<3$ to the diffusion coefficient $|h|^{3}$ in (2).

The inclusion of van der Waals forces has been proposed by several authors $[28,15]$ to describe such physical phenomena as film rupture (in the repulsive case) and the precursor film (in the attractive case). In this paper we only address the attractive case.

The boundary of the support of the weak solution physically corresponds to the contact line, the triple contact point of the air/liquid, air/solid, and solid/liquid interfaces. We denote the respective interfacial surface energies of the three interfaces by $\gamma_{\mathrm{LV}}, \gamma_{\mathrm{SV}}$, and $\gamma_{\mathrm{LS}}$. The spreading parameter, $S$, of the system is

$$
s=\gamma_{S V}-\gamma_{L S}-\gamma_{L V} \text {. }
$$

When $S \geqslant 0$, the drop energetically prefers to completely wet the surface $\dagger$ and the long range character of the molecular interactions are important to the local dynamics close to the liquid/solid interface [22]. Such interactions are important on a mesoscopic length scaleł and can be described in terms of a disjoining pressure [26,20].

Following $[28,10,14]$, the depth averaged fluid velocity satisfies

$$
U(x)=-\frac{h^{2}}{3 \eta}(\Pi(h))_{x}+\frac{\gamma h^{2}}{3 \eta} \frac{\mathrm{d}^{3} h}{\mathrm{~d} x^{3}}=-\frac{A}{6 \pi h^{2} \eta} h_{x}+\frac{\gamma h^{2}}{3 \eta} \frac{\mathrm{d}^{3} h}{\mathrm{~d} x^{3}}
$$

$\dagger$ One calls this situation complete wetting as opposed to partial wetting.

$\ddagger$ Approximateiy 100-1000 A. 
where $\gamma$ is the surface tension and $\eta$ is the fluid viscosity. The disjoining pressure $\Pi$ is taken to be $-\frac{A}{6 \pi h^{3}}$ where $A$ is the Hamaker constant.

Mass conservation gives an evolution equation for the film height:

$$
h_{t}+(h U)_{x}=h_{t}-\left(\frac{A}{6 \pi \eta}(\log h)_{x}-\frac{\gamma h^{3}}{3 \eta} \frac{\mathrm{d}^{3} h}{\mathrm{~d} x^{3}}\right)_{x}=0
$$

In the complete wetting problem, $A>0$. Ignoring the capillary term and considering only the long range van der Waals forces yields a super diffusive equation for the film height

$$
h_{t}=\left(\frac{A}{6 \pi h \eta} h_{x}\right)_{x}=\frac{A}{6 \pi \eta}(\log (h))_{x x} .
$$

Solutions to this problem are known to have quite singular behaviour [12, 13, 24]. In particular, one would expect infinite speed of propagation of the support and it is unclear if one can even make sense of this equation for initial data with compact support.

Several authors have suggested that since intermolecular effects are not expected below a certain length scale, the long range van der Waals model is not reasonable in the limit as $h \rightarrow 0[22,28]$. In this spirit, we propose a simple cut-off of the gradient of the disjoining pressure at a molecular length scale, $h_{m} \dagger$. Mathematically, this means replacing the gradient of the disjoining pressure by

$$
\left[(\Pi(h))_{x}\right]_{c}=\varphi_{c}(h)\left(\frac{-A}{6 \pi h^{3}}\right)_{x}
$$

where $\varphi_{\mathrm{c}}(h)$ is a non-negative cut-off function which is 1 for $h>h_{m}$ and vanishes at $h=0$. We make the ansatz that the cut-off function vanishes like $h^{m}$ as $h \rightarrow 0$. This produces a modified van der Waals term in (5) that is $\sim(\log h)_{x x}$ for $h_{m}<<h<1$ but has a subdiffusive 'porous media' like behaviour, $\left(h^{m}\right)_{x x}$, below the molecular scale $h_{m}$.

The rigorous theorems presented here are for a simplified equation (1) with just the cutoff behaviour. Since the equation with the cut-off van der Waals term (6) is uniformly parabolic in any region where $h \geqslant m>0$, we expect that a suitable 'weak' solution will be completely smooth where $h>0$ and that the regularity and well-posedeness properties of the solution will be controlled by the behaviour of the diffusion coefficients in the limit as $h \rightarrow 0$. For this reason, we expect our results for the simpler model (1) to hold for the full equation with the cut-off van der Waals term (6).

In section 6 we present some computations of both the simplified equation (1) and the full equation (5) with a cut-off van der Waals term (6). In the latter case, the solution exhibits a pronounced 'foot' analogous to the physical precursor film.

\subsection{Degenerate diffusion equations: fourth order versus second order}

We now compare the fourth-order 'lubrication approximation' equation

$$
h_{t}=-\left(|h|^{n} h_{x x x}\right)_{x}=-\left(f(h) h_{x x x}\right)_{x}
$$

to the second-order porous media equation

$$
h_{t}=\left(h^{m}\right)_{x x} \text {. }
$$

1.2.1 The porous media equation For $m>1$, the porous media equation (8) has unique weak solutions. On the real line, if the initial data is non-negative and has compact support, 
then there exists a unique non-negative weak solution to the equation. This solution has compact support that propagates with a finite speed. The porous media equation possesses non-negative self-similar source-type solutions for all $m>1$

$$
\begin{aligned}
& h(x, t)=M^{2 / m} t^{-1 /(m+1)} \Phi\left(x M^{-(m-1) /(m+1)} t^{-1 /(m+1)}\right) \\
& \Phi(y)= \begin{cases}a(m)\left(y_{0}^{2}-y^{2}\right)^{1 /(m-1)} & \text { if } y \leqslant y_{0} \\
0 & \text { if } y>y_{0} .\end{cases}
\end{aligned}
$$

Weak solutions with compact support converge to these source-type solutions as $t \rightarrow \infty$ [21].

Note that for $1<m<2$ the 'source type' solutions 'touch down' with zero slope. In [6] we found a family of solutions for the fourth-order lubrication equation that also touch down with zero slope. In this paper we show that when $1<m<2$ and $n>0$ there exist weak solutions to equation (1) that have zero slope at the edge. The rigorous theory for this second-order problem relies heavily on a maximum or comparison principle. This technique is not directly applicable to higher order problems.

1.2.2. The lubrication approximation The fourth-order problem (7) does not satisfy a maximum principle. However, the equation possesses a number of conserved and dissipated quantities that provide a weak solution theory via energy methods. In particular we have the following weak existence theorem [6]†.

Theorem 1.1. Given any non-negative initial condition $h_{0} \in H^{1}\left(S^{1}\right), h_{0} \geqslant 0$ we have the following results

Case 1. Given $1<n<2,0<s<\min \left(2-n, \frac{1}{2}\right)$, and a time $T$ there exists $h \geqslant 0$, $h \in L^{\infty}\left(0, T ; H^{1}\left(S^{1}\right)\right) \cap L^{2}\left(0, T ; H^{2}\left(S^{1}\right)\right)$, that satisfies the equation in the following sense:

$$
\iint_{Q_{T}} h \varphi_{t}-\iint_{Q_{T}} f(h) h_{x x} \varphi_{x x}-\iint_{Q_{T}} f^{\prime}(h) h_{x} h_{x x} \varphi_{x}=0
$$

Moreover,

$$
\begin{aligned}
& h(x, 0)=h_{0}(x) \quad \forall x \in S^{1} \\
& h_{x}(\cdot, t) \rightarrow h_{0 x} \quad \text { strongly in } L^{2}\left(S^{1}\right) \text { as } t \rightarrow 0 .
\end{aligned}
$$

Furthermore, given $\alpha \geqslant \frac{1}{2}-\frac{s}{4}$, h has the additional regularity

$$
h^{1-s / 2} \in L^{2}\left(0, T ; H^{2}\left(S^{1}\right)\right)
$$

and

$$
\left(h^{\alpha}\right)_{x} \in L^{4}\left(Q_{T}\right)
$$

Moreover, there exist positive $A$ and $c$ such that for all $t \in[0, T]$,

$$
\|h(\cdot, t)-\bar{h}\|_{L^{\infty}} \leqslant A \mathrm{e}^{-c t}
$$

where $\bar{h}$ is the mean value of $h$. A depends only on $\left|h_{0}\right|_{H^{1}}, \bar{h}, n$, and $\left|S^{1}\right|$. The rate of decay, $c$, depends only on $n$, and $\bar{h}$. In particular, if $h_{0}$ is nonzero there exists a time $T^{*}$ after which the solution is a positive strong solution.

$\uparrow$ Similar regularity and long-time results have been recently proven for $0<n<3$ for solutions in a weaker, non-distribution sense [1]. 
Case IA. If $\frac{3}{8}<n \leqslant 1$ the above is true if we replace the equation (10) with a solution in the sense

$$
\iint_{Q_{T}} h \varphi_{t}-\iint_{Q_{T}} f(h) h_{x x} \varphi_{x x}-\iint_{Q_{T}} n h^{n-\alpha}\left(\frac{h^{\alpha}}{\alpha}\right)_{x} h_{x x} \varphi_{x}=0
$$

where $\alpha$ is chosen so that $n>\alpha \geqslant \frac{1}{2}-\frac{s}{4}$.

Case 2. If $2<n<3$, given any $0<r<1$ satisfying $0<2+r-n<1$ then for any $T$ there exists $h \geqslant 0$ such that $h \in L^{\infty}\left(0, T ; H^{1}\left(S^{1}\right)\right)$ and $h$ satisfies the equation in the following sense:

$\iint_{Q_{T}} h \varphi_{1}+\frac{3}{2} \iint_{Q_{T}} f^{\prime}(h) h_{x}^{2} \varphi_{x x}+\frac{1}{2} \iint_{Q_{T}} f^{\prime \prime}(h) h_{x}^{3} \varphi_{x}+\iint_{Q_{T}} f(h) h_{x} \varphi_{x x x}=0$.

The initial data are achieved as above. Furthermore, $h$ has the additional regularity

$$
h^{1+r / 2} \in L^{2}\left(0, T ; H H^{2}\left(S^{1}\right)\right)
$$

and

$$
\left(h^{\alpha}\right)_{x} \in L^{4}\left(Q_{T}\right) \quad \forall \alpha \geqslant \frac{r}{4}+\frac{1}{2} .
$$

The long time behaviour is as above (11).

The statement for $n=2$ is as in case 2 with a minor change in the form of the equation. There also is an existence result for $0<n<3 / 8$ in a weaker sense introduced in [2]. See [6] for a discussion.

What is striking about these results is that the additional regularity of the weak solutions for $0<n<3$ is in exact agreement with the regularity of 'zero contact angle' non-negative source type solutions (see (18)-(20) below). That is, if we assume that the limiting solution $h(x, t)$ has support compactly contained in $S^{1}$ and and $h(x) \sim x^{\beta}$ at the edge of the support for all $t$ on some interval $[0, T]$, then the regularity constraints demand that

$$
\begin{array}{ll}
\beta \geqslant 2 & 0<n<3 / 2 \\
\beta \geqslant 3 / n & 3 / 2<n<3 .
\end{array}
$$

Furthermore, the techniques used in proving theorem 1.2 fail for $n \geqslant 3$. This is consistent with the lack of similarity or advancing front solutions for $n \geqslant 3$.

We briefly discuss some exact solutions for the equation

$$
h_{t}+\left(|h|^{n} h_{x x x}\right)_{x}=0 \text {. }
$$

Compactly supported non-negative self-similar 'source type' solutions exist for all $0<n<3$ [3]. They have the scaling form

$$
h(x, t)=t^{-\alpha} H(\eta) \quad \eta=x t^{-\alpha} \quad \alpha=\frac{1}{n+4} .
$$

Where $H(\eta)$ solves the ODE

$$
H^{n} H_{\eta \eta \eta}=\alpha \eta H \text {. }
$$

For a given $n$ and mass, there is more than one compactly supported non-negative symmetric solution to this ODE. However, if we impose the additional constraint that the solution have $H_{\eta}=0$ at the edge of the support, the solution is unique. This was proven in [3], in which 
they also proved that these 'most regular' solutions have the following behaviour at the edge of their support: Let $[-a, a]$ denote the support of $H(\eta)$. Then

$$
\begin{aligned}
& \text { for } 0<n<3 / 2, \quad H(\eta) \sim(a-\eta)^{2} \text { as } \eta \uparrow a \\
& \text { for } n=3 / 2 H(\eta) \sim(a-\eta)^{2} \log (1 /(a-\eta))^{2 / 3} \text { as } \eta \uparrow a \\
& \text { for } 3 / 2<n<3 H(\eta) \sim(a-\eta)^{3 / n} \text { as } \eta \uparrow a .
\end{aligned}
$$

The less regular solutions have $H(\eta) \sim(a-\eta)$.

Starov [27] first noted that there are no finite mass 'source-type' solutions for $n=3$. Brenner and Bertozzi [9] addressed the significance of this fact for the physical problem of spreading droplets. The $n=3$ case arises when there is a no-slip boundary condition at the liquid/solid interface. The lack of such scaling solutions is consistent with the fact that a no-slip boundary condition leads to infinite energy dissipation at the contact line for spreading drops with a finite contact angle [17, 19].

The non-existence of source type solutions for $n \geqslant 3$ is due to the structure of the ODE (17) and is in sharp contrast to the source type solutions for the porous media equation (8) which exist for all $m>1$.

There are also travelling wave solutions of the form $h(x, t)=H(x-c t)$ as described in [8]. Again, we see transitions in the behaviour at critical values of $n$. It is noteworthy that there are no advancing front solutions for $n \geqslant 3$. For $3 / 2<n<3$ there are advancing front solutions with the simple form

$$
h(x, t)=\left\{\begin{array}{ll}
A(x-c t)^{3 / n} & x>c t \\
0 & \text { otherwise }
\end{array} \quad c=\left(\frac{3}{n}-2\right)\left(\frac{3}{n}-1\right) \frac{3}{n} A^{n} .\right.
$$

For $0<n<3 / 2$ there are advancing front solutions with quadratic $A(x-c t)^{2}$ leading order behaviour [8]. Finally, there are exact steady solutions for all $n$

$$
h(x, t)= \begin{cases}A-B x^{2} & |x|<A / \sqrt{B} \\ 0 & \text { otherwise. }\end{cases}
$$

The proof of the theorem 1.1 depends on certain dissipated energies. In this paper we derive analogous estimates for the equation with both fourth-order and second-order terms present:

$$
\frac{\mathrm{d} h}{\mathrm{~d} t}+\left(h^{n} h_{x x x}\right)_{x}-\left(h^{m}\right)_{x x}=0 .
$$

We briefly discuss some properties of smooth solutions that we use to prove needed a priori estimates.

For example, we have conservation of mass,

$$
\int_{S^{1}} h(x, t) \mathrm{d} x=\int_{S^{1}} h_{0}(x) \mathrm{d} x .
$$

When $1<m<2$, we have dissipation of surface tension energy

$$
\begin{aligned}
\int_{S^{1}}\left|h_{x}(x, T)\right|^{2} \mathrm{~d} x+\iint_{Q_{T}} f(h) h_{x x x}^{2}+m \iint_{Q_{T}} h^{m-1} h_{x x}^{2} \\
+\frac{m(m-1)(2-m)}{3} \iint_{Q_{T}} h^{m-3} h_{x}^{4} \mathrm{~d} x \mathrm{~d} t=\int_{S^{1}}\left|h_{x}(x, 0)\right|^{2} \mathrm{~d} x
\end{aligned}
$$


and the basic entropy dissipation: consider a function $G(y)$ satisfying $G^{\prime \prime}(y)=1 / f(y)$ for $y>0$. The convexity of $G$ and mass conservation allow us to choose $G$ so that $\int_{S^{1}} G(h(x, t)) \mathrm{d} x \geqslant 0$ for all $t$. Integration by parts yields

$\int_{S^{1}} G(h(x, T)) \mathrm{d} x+\iint_{Q_{T}} h_{x x}^{2}+m \iint_{Q_{T}} \frac{h^{m-1}}{f(y)} h_{x}^{2} \mathrm{~d} x \mathrm{~d} t=\int_{S^{1}} G(h(x, 0)) \mathrm{d} x$.

For $n=0$, the linear problem, the entropy is merely the $L^{2}$ norm. Bernis and Friedman first introduced these entropies in [2]. In a previous work [6], we used these and a new class of entropies to prove theorem 1.1. We show that for $1<m<2$ the same results hold and we extend results to all $n>0$ by exploiting sharper estimates obtained by the presence of the second-order term. For $n>3 / 2$, we obtain sharp regularity results. We now discuss this briefly:

1.2.3. Competition between the fourth-and second-order terms It is natural to expect that either the second-order term or the fourth-order term will control the dynamics at the edge of the support of the solution. "In section 2 we discuss leading-order asymptotics for the solution near the edge of its support. For $n \geqslant 3$ and $1<m<2$, the asymptotics predict that locally there are advancing travelling wave solutions and that their behaviour at the edge of the support is dictated by the second-order term. That is, the solution at the edge $\sim(x-x(t))^{1 /(m-1)}$. Moreover, for $3 / 2<n<3$, and $1<m<2$, whichever term gives higher regularity at the edge will dominate. We also discuss some expansions for the case $n<3 / 2$. The predictions and possibilities are summarized in the chart below. The case $n=3 / 2$ is special as log dependences play a role when the fourth-order term dominates at the edge 19.

Table 1. Behaviour at the edge: second versus fourth order.

\begin{tabular}{|c|c|c|c|c|}
\hline term & behaviour at edge & $0<n<3 / 2$ & $3 / 2<n<3$ & $n \geqslant 3$ \\
\hline $\begin{array}{l}\left(h^{n} h_{x x x}\right)_{x} \\
n>0\end{array}$ & $\begin{array}{l}\left(x-x_{0}(t)\right)^{2} \text { for } 0<n<3 / 2 \\
\left(x-x_{0}(t)\right)^{3 / n} \text { for } 3 / 2<n<3 \\
\text { no solutions for } n \geqslant 3\end{array}$ & $\begin{array}{l}\text { can control } \\
\text { edge if } m>n\end{array}$ & $\begin{array}{l}\text { controis edge if } \\
1 /(m-1)<3 / n\end{array}$ & no solutions \\
\hline $\begin{array}{l}\left(h^{m}\right)_{x x} \\
1<m<2\end{array}$ & $\left(x-x_{0}(t)\right)^{1 /(m-1)}$ & $\begin{array}{l}\text { can control edge } \\
\text { if } 1 /(m-1)>3 / n\end{array}$ & $\begin{array}{l}\text { controls edge if } \\
1 /(m-1)>3 / n\end{array}$ & $\begin{array}{l}\text { always controls } \\
\text { edge }\end{array}$ \\
\hline
\end{tabular}

\subsection{Main results}

Given that the techniques used to prove theorem 1.1 break down as $n \rightarrow 3$, a natural question is what can one say about the problem for $n \geqslant 3$. One result of this paper is that the inclusion of a second-order 'porous media' term with $1<m<2$ enables us to prove the same results as in theorem 1.1 . We derive a weak existence theory for the equation

$$
h_{t}+\left(h^{n} h_{x x x}\right)_{x}-\left(h^{m}\right)_{x x}=0
$$

for $n>0$ and $1<m<2$. In particular, for $n>3 / 2$, the regularity of the weak solution is in sharp agreement with that predicted by asymptotics at the edge of the support of the solution (see table 1 and section 2). Thus the existence theorem supports the predictions made by the asymptotics.

The main theorem is 
Theorem 1.2. Given any non-negative initial condition $h_{0} \in H^{1}\left(S^{1}\right), h_{0} \geqslant 0$ we have the following results

Case 1. Let $n \geqslant 3,1<m<2$. Then for any time $T$, there exists $h$ such that $h \in L^{\infty}\left(0, T ; H^{1}\left(S^{1}\right)\right), h \geqslant 0$, and $h$ satisfies the equation in the following sense of distributions:

$$
\begin{gathered}
\iint_{Q T} h \varphi_{t}+\frac{3}{2} \iint_{Q_{T}} f^{\prime}(h) h_{x}^{2} \varphi_{x x}+\frac{1}{2} \iint_{Q_{T}} f^{\prime \prime}(h) h_{x}^{3} \varphi_{x}+\iint_{Q T} f(h) h_{x} \varphi_{x x x} \\
=\iint_{Q_{T}}\left(h^{m}\right)_{x} \varphi_{x}
\end{gathered}
$$

The initial data are achieved as in theorem 1.1. Furthermore given $\alpha_{0}>0$ there exists a solution $h$ satisfying the above that has the additional regularity

$$
\begin{aligned}
& h^{\frac{m+1}{2}} \in L^{2}\left(0, T ; H^{2}\left(S^{1}\right)\right) \\
& \left(h^{\gamma}\right)_{x}, \in L^{4}\left(Q_{T}\right) \quad \text { for all } \gamma \geqslant \frac{m+1}{4} \\
& \left(h^{(\alpha+m-1) / 2}\right)_{x}, \in L^{2}\left(Q_{T}\right) \quad \text { for all } \alpha \geqslant \alpha_{0}
\end{aligned}
$$

and the long time behaviour

$$
\|h(x, t)-\bar{h}\|_{L^{\infty}} \leqslant A \mathrm{e}^{-c t} \text {. }
$$

$A$ is determined by $\left|h_{0}\right|_{H^{1}}, n, \bar{h}$, and $\left|S^{1}\right|$. The rate of decay, $c$, is determined by $n$ and $\bar{h}$. In particular, if $h_{0}$ is nonzero there exists a critical time $T^{*}$ after which the solution is guaranteed to be strong and positive.

Case 2. For $2<n<3,1<m<2$ the above existence and long time result is true. Moreover, given $r$ satisfying both $0<r<1$ and $0<2+r-n$, there exists $h$ with the additional regularity

$$
\begin{aligned}
& h^{1+r / 2} \in L^{2}\left(0, T ; H^{2}\left(S^{1}\right)\right), \\
& \left(h^{\alpha}\right)_{x} \in L^{4}\left(Q_{T}\right) \quad \forall \alpha \geqslant \frac{r+2}{4} .
\end{aligned}
$$

Case 3. Let $1<n<2,1<m<2$. Then on any time interval $[0, T]$ given any $0<s<\min \left(2-n, \frac{1}{2}\right)$ and any $\alpha_{0}>\max (0, m-n+1)$, there exists a solution in the following sense of distributions:

$$
\iint_{Q_{T}} h \varphi_{t}-\iint_{Q_{T}} f(h) h_{x x} \varphi_{x x}-\iint_{Q_{T}} f^{\prime}(h) h_{x x} h_{x} \varphi_{x}=\iint_{Q_{T}}\left(h^{m}\right)_{x} \varphi_{x} .
$$

The solution has the regularity

$$
\begin{aligned}
& h^{1-s / 2} \in L^{2}\left(0, T ; H^{2}\left(S^{1}\right)\right) \\
& \left(h^{\beta}\right)_{x} \in L^{4}\left(Q_{T}\right) \quad \forall \beta \geqslant \frac{-s+2}{4} \\
& \left(h^{\alpha}\right)_{x} \in L^{2}\left(Q_{T}\right) \quad \forall \alpha \geqslant \alpha_{0} \\
& \left(h^{(1-n-s+m) / 2}\right)_{x}, \in L^{2}\left(Q_{T}\right) \\
& h_{x} \in L^{\infty}\left(0, T ; L^{2}\left(S^{1}\right)\right) .
\end{aligned}
$$

and the long time behaviour as above. 
The theorem for $n=2$ is as in case 2 , with a minor change in the definition of weak solution. The reader can derive results for $0<n \leqslant 1$ following the proofs in [6].

Significant remark. Note that if we make the ansatz that the weak solution above has a local power law $\left(x-x_{0}(t)\right)^{\beta}$ where $x_{0}(t)$ is the edge of the support then the additional regularity in the statement of the theorem implies

$$
\beta \geqslant \begin{cases}\max (3 / n, 1 /(m-1)) & n>3 / 2 \\ \max \left(2,1 /\left(\frac{1}{2}+m-n\right)\right) & 0<n<3 / 2 .\end{cases}
$$

In particular, the theorem is sharp given the asymptotics for $n>3 / 2$.

The techniques are similar to [6]. We introduce convex entropies to prove the existence and long time result. For $n \geqslant 3$ existence and decay of weak solutions follows directly from energy dissipation. The convex entropies are only needed for additional regularity. As in [6], we use weak convergence arguments for nonlinear functions of $h$ in Sobolev spaces to prove existence. There is a direct relation between the exponents $m$ and $n$ that allow for such spreading solutions.

We use a regularization introduced in [2] and used in [6]. In section 6 we present some numerical calculations of the solutions which demonstrate the predicted interplay between the 'lubrication approximation' term and the 'porous media' term. The simulations indicate that the support of the solution has finite speed of propagation and continuous flux, two properties desirable for a physically correct model.

The paper is organized as follows. Section 2 provides a discussion of the asymptotics at the edge of the solution as a motivation for the sharpness of the theorems. Section 3 reviews the properties of the regularization scheme. Section 4 proves the existence results for nonnegative initial data for $0<n$. Section 5 proves the long time results for non-negative initial data for $0<n$. Section 6 presents numerical calculations. Section 7 summarizes this paper and discusses unsolved problems.

\section{Competition between second- and fourth-order terms: asymptotics at the edge of the support}

Before proceeding with the rigorous analysis, we use asymptotics to study the competition between the second- and fourth-order terms at the edge of the support.

Let us assume that we have a weak solution to the equation

$$
h_{t}=-\left(h^{n} h_{x x x}\right)_{x}+\left(h^{m}\right)_{x x}
$$

with compact support the edge of which propagates with finite speed. Either the fourth- or the second-order term determines the motion of the edge of the support. In this section we present a simple asymptotic argument for the competition between the two terms in terms of $n$ and $m$. In section 4 we present rigorous results which confirm the predictions made by the asymptotics. For $n>3 / 2$, the rigorous analysis is sharp in that the regularity of the weak solution is in exact agreement with that predicted by the asymptotics.

We recall that for the lubrication approximation equation (7) advancing fronts only exist for $0<n<3[3,8]$. This suggests that for (24) to have an advancing front for $n \geqslant 3$, the motion must be due to solely the second-order 'porous media' term. However, when $n<3$ it should be possible to have either the second or fourth-order term determine the motion of the edge of the support. The leading order asymptotics below indicate that this intuition is reasonable. 
A standard technique is to assume that locally the solution looks like a travelling wave near the edge of its support. Hence we consider solutions of the form

$$
h(x, t)=H(x-c t) .
$$

This yields the following equation for $H$

$$
-c H^{\prime}(\eta)=-\left(H^{n}(\eta) H^{\prime \prime \prime}(\eta)\right)^{\prime}+\left(H^{m}(\eta)\right)^{\prime \prime}
$$

where $\eta=x-c t$. Integrating once, we find

$$
A-c H(\eta)=-H^{n}(\eta) H^{\prime \prime \prime}(\eta)+\left(H^{m}(\eta)\right)^{\prime} .
$$

$A$ is determined by the regularity at the edge of the support of $H$. Without loss of generality, the edge of the support of the solution corresponds to $\eta=0$.

There are four cases to consider: $0<n<3 / 2$ and $3 / 2<n$ and for each of these either $A=0$ or $A \neq 0$. For technical reasons, we do not consider $n=3 / 2$ as the expected behaviour is not purely algebraic (19).

Case 1. $3 / 2<n$

Case 1a. $A=0$. Assume that $H$ touches down like $\eta^{\theta}$, with $\theta \neq 1,2$. Then

$$
H^{n} H^{\prime \prime \prime} \sim \eta^{n \theta+\theta-3} \quad\left(H^{m}\right)^{\prime} \sim \eta^{m \theta-1} .
$$

If the porous media term is the lowest-order term on the right-hand side of (25) we have $H \sim\left(H^{m}\right)^{\prime}$ therefore

$$
\eta^{\theta} \sim \eta^{m \theta-1} \quad \Longrightarrow \quad \theta=\frac{1}{m-1} \text {. }
$$

$\theta=1 /(m-1)$ is the generic case for solutions of the porous media equation. Note that touchdown with zero slope at the edge implies $\theta>1$ which gives the constraint $1<m<2$. For this behaviour to dominate the fourth-order lubrication term we require

$$
H^{n}(\eta) H^{\prime \prime \prime}(\eta) \prec\left(H^{m}(\eta)\right)^{\prime} \quad \Longrightarrow \quad \eta^{(n+1) \theta-3} \prec \eta^{\theta m-1} \quad \Longrightarrow \quad \theta>\frac{2}{n+1-m} .
$$

Combining these, we see that the porous media term dominates at the edge of the support whenever

$$
\frac{1}{m-1}=\theta>\frac{2}{n+1-m} \Rightarrow \frac{3}{n}<\frac{1}{m-1} .
$$

The same argument shows that if the lubrication approximation term is the lowest-order term then

$$
\theta=\frac{3}{n} \quad \text { and } \quad \frac{1}{m-1}<\frac{3}{n} .
$$

If $\theta=3 / n=1 /(m-1)$ the two terms are of equal importance.

This argument suggests that the equation selects the power law that gives greater regularity at the edge. In section 4 , we prove that for $3 / 2<n$ and $1<m<2$ the regularity of the weak solutions is in sharp agreement with the asymptotics (26)-(27).

Case $1 b . A \neq 0$. In this case, if the porous media term is the lowest-order term then $A \sim\left(H^{m}\right)^{\prime}$. As above, this implies

$$
\theta=\frac{1}{m} \quad \text { and } \quad \frac{3}{n+1}<\frac{1}{m} \text {. }
$$


If the lubrication term is lowest-order then

$$
\theta=\frac{3}{n+1} \quad \text { and } \quad \frac{1}{m}<\frac{3}{n+1} .
$$

For $n>3 / 2$ and $1<m<2$, the existence theory of section 4 excludes behaviours (28)-(29) as they are not sufficiently regular.

Case 2. $0<n<3 / 2$

Case $2 a . A \neq 0$. Again, the regularity of the weak solutions rules out this case.

Case $2 b . A=0$. We recall that for $0<n<3 / 2$ the lubrication approximation equation (7) has non-negative self-similar source-type solutions with expanding support with $\theta=2$ [3] and receding travelling wave solutions with $\theta=3 / n$ [8]. Therefore the options are: the front could recede with $\theta=3 / n$ or the front could advance with $\theta=2$ (lubrication dominated) or $\theta=1 /(m-1)$ (porous media dominated). Since we are ultimately concerned with the advancing front case $\dagger$ we consider only $\theta=2$ for the lubrication dominated behaviour.

In the case $\theta=2$, the lubrication approximation term dominates the motion of the edge, and we seek an expansion

$$
H(\eta)=k \eta^{2}+A \eta^{a}+B \eta^{b}+C \eta^{c}+\ldots
$$

where $2<a<b<c<\ldots$. Therefore

$$
H^{n}(\eta)=k^{n} \eta^{2 n}+n k^{n-1} \eta^{2(n-1)}\left(A \eta^{a}+B \eta^{b}+\ldots\right)+n(n-1) k^{n-2} \eta^{2(n-2)}(\ldots)^{2}+\ldots
$$

$H^{m-1}(\eta)$ is expanded similarly. Defining $\tilde{A}=a(a-1)(a-2) A, \tilde{B}=b(b-1)(b-2) B$, $\tilde{C}=c(c-1)(c-2) C$, we find

$$
\begin{aligned}
-H^{n} H^{\prime \prime \prime}+H^{m-1} H^{\prime}= & \\
& -\left(k^{n} \eta^{2 n}+n A k^{n-1} \eta^{2 n+a-2}+n B k^{n-1} \eta^{2 n+b-2}+n C k^{n-1} \eta^{2 n+c-2}+\ldots\right) \\
& \times\left(\tilde{A} \eta^{a-3}+\tilde{B} \eta^{b-3}+\tilde{C} \eta^{c-3}+\ldots\right) \\
& +\left(k^{m-1} \eta^{2 m-2}+A(m-1) k^{m-2} \eta^{2 m+a-4}+B(m-1) k^{m-2} \eta^{2 m+b-4}+\ldots\right) \\
& \times\left(2 k \eta+a A \eta^{a-1}+b B \eta^{b-1}+c C \eta^{c-1} \ldots\right) \\
& =\left(-\tilde{A} k^{n} \eta^{2 n+a-3}-\tilde{B} k^{n} \eta^{2 n+b-3}-\tilde{C} k^{n} \eta^{2 n+c-3}-\ldots\right) \\
& +\left(2 k^{m} \eta^{2 m-1}+a A k^{m-1} \eta^{2 m+a-3}+b B k^{m-1} \eta^{2 m+b-3}+\ldots\right) .
\end{aligned}
$$

Recall that $-c H=-H^{n} H^{\prime \prime \prime}+H^{m-1} H^{\prime}$, hence (30) implies the lowest-order term above must be $\eta^{2}$. We now begin considering the possibilities.

Case 1. No lowest-order terms cancel. In this case, the lowest-order term from the lubrication term is the term of order 2: $\eta^{2 n+a-3} \sim \eta^{2}$. This implies $a=5-2 n$. The coefficient of this term determines the speed of the edge. The assumption $a>2$ then imposes the requirement $n<3 / 2$. The fact that the lowest-order term comes from the lubrication approximation term implies $\eta^{2 n+a-3} \preceq \eta^{2 m-1}$, implying $2 n+a-3 \leqslant 2 m-1$, hence $m \geqslant 3 / 2$. This suggests that if $m$ and $n$ satisfy

$$
n<\frac{3}{2} \quad m \geqslant \frac{3}{2}
$$

$\dagger$ Our existence theorem states that the weak solution will eventually become a strong positive solution so that the support must eventually increase to fill the whole periodic domain. 
then there is an expansion of the form $H(\eta)=k \eta^{2}+A \eta^{5-2 n}+\ldots$, where $2 k$ determines the second derivative at the edge and $A$ determines the speed of the edge.

Case 2. The lowest-order term from the lubrication term cancels with the lowest-order term from the porous medium term. If these two terms are to cancel, we need $\eta^{2 n+a-3} \sim \eta^{2 m-1}$. This determines $a=2(m-n+1)$ and $A$ is then determined by $a$ and $k: a(a-1)(a-2) A k^{n}=$ $2 k^{m}$. The requirement $2<a$ implies $m>n$. The cancellation makes $\eta^{2 n+b-3}$ the lowest-order term from the lubrication term, and $\eta^{2 m+a-3}$ the lowest-order term from the porous medium term. Since the lubrication term dominates the behaviour, $\eta^{2 n+b-3} \sim \eta^{2}$, determining $b=5-2 n$. The requirement $a<b$ implies $m<3 / 2,2<b$ implies $n<3 / 2$. Moreover, $\eta^{2 n+b-3} \preceq \eta^{2 m+a-3}$ implies $m \geqslant n / 2+3 / 4$, which immediately implies $m>n$. This suggests that if $m$ and $n$ satisfy

$$
n<\frac{3}{2} \quad m<\frac{3}{2} \quad m \geqslant \frac{1}{2} n+\frac{3}{4}
$$

then there is an expansion of the form $H(\eta)=k \eta^{2}+A \eta^{2 m-2 n+2}+B \eta^{5-2 n}+\ldots$, where $k$ determines $A$ and the second derivative at the edge and $B$ determines the speed of the edge.

Case 3. The lowest-order and second lowest-order terms from the lubrication term cancel with the respective terms from the porous media term. Repeating the above argument, we find that $a$ and $b$ are determined by $m$ and $n$, and if $m$ and $n$ satisfy

$$
n<\frac{3}{2} \quad m<\frac{1}{2} n+\frac{3}{4} \quad m \geqslant \frac{1}{3} n+1
$$

then there is an expansion of the form $H(\eta)=k \eta^{2}+A \eta^{2 m-2 n+2}+B \eta^{4 m-4 n+2}+C \eta^{5-2 n}+\ldots$ Here $k$ determines $A, B$, and the second derivative at the edge, while $C$ determines the speed of the edge.

We note that in the above, we have assumed that the lowest-order terms in the expansion of $H^{n}(2)$ have been from $\eta^{2(n-1)}\left(A \eta^{a}+\ldots\right)$ and not from $\eta^{2(n-2)}\left(A \eta^{a}+\ldots\right)^{2}$ or other terms. Under this assumption, we can continue the above process indefinitely, and we see that for any $m$ and $n$ satisfying $n<3 / 2$ and $m>n$, there is an expansion $H$ so that $H(\eta)=k \eta^{2}+\ldots+\tilde{c} \eta^{5-2 n}+\ldots$, where $2 k$ determines the second derivative at the edge and the coefficients of the intermediate terms and $\tilde{c}$ determines the speed. This suggests that there are two degrees of freedom: the second derivative and the speed. A more in depth study of the asymptotics in which an inner expansion at the edge is matched to an outer expansion away from the edge may yield additional matching constraints.

Thus it is possible to have leading order $\eta^{2}$ behaviour when $m>n$ and from the above arguments for $n>3 / 2$, one can have $\eta^{1 /(m-1)}$ behaviour when $1 /(m-1)>3 / n$. Since $n>m$ and $n<3 / 2$ always implies $1 /(m-1)>3 / n$, there is always at least one possible behaviour for any $0<n<3 / 2,1<m<2$.

\section{Regularized problem}

To prove existence of weak solutions and to numerically compute the weak solutions we use a regularization scheme introduced in [2] and used in $[6,1]$.

The regularization involves altering the equation and lifting the initial data. We bound the initial data for the regularized problem away from zero by

$$
h_{\epsilon 0}(x)=h_{0}(x)+\delta(\epsilon) \text {. }
$$

where $\delta(\epsilon)>0$ and $h_{\epsilon 0} \rightarrow h_{0}$ in $H^{1}\left(S^{1}\right)$ as $\epsilon \rightarrow 0$. 
In addition, we regularize the equation by considering

$$
\begin{aligned}
& h_{\epsilon t}=-\left(f_{\epsilon}\left(h_{\epsilon}\right) h_{\epsilon x x x}\right)_{x}+\left(h_{\epsilon}{ }^{m}\right)_{x x} \\
& f_{\epsilon}\left(h_{\epsilon}\right)=\frac{h_{\epsilon}{ }^{4}\left|h_{\epsilon}\right|^{n}}{\epsilon\left|h_{\epsilon}\right|^{n}+h_{\epsilon}{ }^{4}} .
\end{aligned}
$$

We remark that $f_{\epsilon}$ is still degenerate; however for $n<4, f_{\epsilon}(y) \sim y^{4} / \epsilon$ as $y \rightarrow 0$. This degree of degeneracy is more tractable and this approximate problem has unique positive smooth solutions for all time.

Theorem 3.1. (Global existence of unique smooth positive solutions for the regularized problem.) Let $h_{0} \in H^{1}\left(S^{1}\right), h_{0} \geqslant 0,1<m<2$. Given an initial condition

$$
h_{\epsilon 0}(x)=h_{0}(x)+\delta(\epsilon)
$$

there exists a unique positive solution to the regularized equation

$$
\begin{aligned}
& h_{\epsilon t}=-\left(f_{\epsilon}\left(h_{\epsilon}\right) h_{\epsilon x x x}\right)_{x}+\left(h_{\epsilon}{ }^{m}\right)_{x x} \\
& f_{\epsilon}\left(h_{\epsilon}\right)=\frac{h_{\epsilon}{ }^{4}\left|h_{\epsilon}\right|^{n}}{\epsilon\left|h_{\epsilon}\right|^{n}+h_{\epsilon}{ }^{4}} .
\end{aligned}
$$

The proof is a minor modification of that presented in [2], and summarized in [6]. For this reason, we omit many details. The main points are :

- Classical parabolic Schauder estimates guarantee existence of a smooth solution up to a time $\sigma$.

- In this short time of existence, for any $t<\sigma$, the smooth solution satisfies

$$
\begin{aligned}
\int_{S^{1}} h_{\epsilon_{x}}^{2}(x, t) \mathrm{d} x & +\int_{0}^{t} \int_{S^{1}} f_{\epsilon}\left(h_{\epsilon}\right) h_{\epsilon x x x}^{2} \\
& +\frac{m(m-1)(2-m)}{3}\left(\frac{4}{m+1}\right)^{4} \int_{0}^{t} \int_{S^{1}}\left(h_{\epsilon}{ }^{\frac{m+1}{4}}\right)_{x}^{4}+m \int_{0}^{t} \int_{S^{1}} h_{\epsilon}^{m-1} h_{\xi_{x x}}^{2} \\
& =\int_{S^{1}} h_{\epsilon x}^{2}(x, 0) \mathrm{d} x .
\end{aligned}
$$

Since $h_{\epsilon 0} \rightarrow h_{0}$ in $H^{1}\left(S^{1}\right)$, (31) provides an a priori upper bound for $\int h_{\epsilon x}^{2}$ independent of $t$ and $\epsilon$ at any time $t<\sigma$. This in turn yields an a priori bound for the Hölder norm $\left|h_{\epsilon}\right|_{C^{1 / 2}\left(S^{1}\right)}$

$$
\begin{aligned}
& \left|h_{\epsilon}\left(x_{1}, t\right)-h_{\epsilon}\left(x_{2}, t\right)\right| \leqslant C\left|x_{1}-x_{2}\right|^{1 / 2} \quad \forall t<\sigma, \\
& \left|h_{\epsilon}(\cdot, t)\right|_{L^{\infty}\left(S^{1}\right)} \leqslant C \quad \forall t<\sigma .
\end{aligned}
$$

Equations (31)-(33) imply

$$
\left|h_{\epsilon}\left(x, t_{1}\right)-h_{\epsilon}\left(x, t_{2}\right)\right| \leqslant C\left|t_{1}-t_{2}\right|^{1 / 8} .
$$

In all of the above, $C$ depends only on the $H^{1}$ norm of the initial data, $\left|h_{0}\right|_{H^{1}}$.

- We introduce the convex 'entropy'

$$
G_{0}^{\prime \prime}(y)=\frac{1}{y^{n}} \quad \text { for } y>0 .
$$


Integration by parts yields

$$
\begin{aligned}
\int_{S^{1}} \frac{\epsilon}{6 h_{\epsilon}(x, t)^{2}} \mathrm{~d} x & +\int_{S^{1}} G_{0}\left(h_{\epsilon}(x, t)\right) \mathrm{d} x+\int_{0}^{t} \int_{S^{1}} h_{\epsilon x}^{2} \\
& +m \int_{0}^{t} \int_{S^{1}} \frac{h_{\epsilon}^{m-1}}{f_{\epsilon}\left(h_{\epsilon}\right)} h_{\epsilon x}^{2}=\int_{S^{1}} \frac{\epsilon}{6 h_{\epsilon 0}(x)^{2}}+G_{0}\left(h_{\epsilon 0}(x)\right) \mathrm{d} x \leqslant C_{\epsilon}
\end{aligned}
$$

for any $t<\sigma$. As in [2] and [6], this a priori bound for $\int_{S^{1}} \epsilon / h_{\epsilon}^{2}$ and the Hölder continuity (32) provide an a priori pointwise lower bound for the solution $h_{\epsilon}(x, t)$.

- The a priori bound for the minimum depends on $\epsilon$ and $\left|h_{0}\right|_{k^{1}}$ and the bound for the maximum depends only on $\left|h_{0}\right|_{k^{1}}$. Hence the solution is uniformly parabolic on $[0, \sigma]$ and can be continued to any time $T$.

- Uniqueness follows from energy methods, as in [2].

We remark that (32)-(34) imply that $\left\{h_{\epsilon}\right\}$ is a uniformly bounded equicontinuous family of functions on $Q_{T}$. The Arzela-Ascoli theorem guarantees the existence of a subsequence that converges uniformly to a limit, $h$.

\section{Existence of weak solutions}

We recall the regularized equation and initial data from the previous section:

$$
\begin{aligned}
& h_{\epsilon 0}(x)=h_{0}(x)+\delta(\epsilon) \\
& h_{\epsilon t}=-\left(f_{\epsilon}\left(h_{\epsilon}\right) h_{\epsilon x x x}\right)_{x}+\left(h_{\epsilon}{ }^{m}\right)_{x x} \\
& f_{\epsilon}\left(h_{\epsilon}\right)=\frac{h_{\epsilon}{ }^{4}\left|h_{\epsilon}\right|^{n}}{\epsilon\left|h_{\epsilon}\right|^{n}+h_{\epsilon}{ }^{4}} .
\end{aligned}
$$

Let

$$
Q_{T}=S^{1} \times(0, T) \quad \varphi \in C_{0}^{\infty}\left(0, T ; C^{\infty}\left(S^{1}\right)\right)
$$

Recall the energy dissipation (31)

$$
\int_{S^{1}} h_{\epsilon x}^{2}(x, t) d x \leqslant \int_{S^{1}} h_{\epsilon 0 x}^{2} d x=C .
$$

The Sobolev embedding theorem implies there exists an $M<\infty$ such that

$$
\left|h_{\epsilon}(x, t)\right| \leqslant M \quad \forall x, t .
$$

In all of the following theorems, the initial data are achieved as in theorem 1.2†.

Proposition 4.1. Given any non-negative initial condition $h_{0} \in H^{1}\left(S^{1}\right), h_{0} \geqslant 0$ we have the following results

Case 1. Let $n \geqslant 3,1<m<2$, and let $h_{\epsilon}$ be the unique positive smooth solution to the regularized problem (36)-(38) with $\delta(\epsilon)$ chosen so that $\delta(\epsilon) \rightarrow 0$ as $\epsilon \rightarrow 0$. Then on any time interval $[0, T]$, there exists a subsequence that converges pointwise uniformly and weakly in $L^{\infty}\left(0, T ; H^{1}\left(S^{1}\right)\right)$ to a non-negative $h$ and $h$ satisfies the equation in the following sense:

$$
\begin{gathered}
\iint_{Q_{T}} h \varphi_{t}+\frac{3}{2} \iint_{Q_{T}} f^{\prime}(h) h_{x}^{2} \varphi_{x x}+\frac{1}{2} \iint_{Q_{T}} f^{\prime \prime}(h) h_{x}^{3} \varphi_{x}+\iint_{Q_{T}} f(h) h_{x} \varphi_{x x x} \\
=\iint_{Q_{T}}\left(h^{m}\right)_{x} \varphi_{x} .
\end{gathered}
$$

$\dagger$ The proof of this is standard, and we refer the reader to [2] for further details. 
The initial data is achieved as in theorem 1.l. Furthermore, given $\alpha_{0}>0$, there exists $h$ with the additional regularity

$$
\begin{aligned}
& h^{\frac{m+1}{2}} \in L^{2}\left(0, T ; H^{2}\left(S^{1}\right)\right) \\
& \left(h^{\gamma}\right)_{x} \in L^{4}\left(Q_{T}\right) \quad \forall \gamma \geqslant \frac{m+1}{4} . \\
& \left(h^{\frac{\alpha+m-1}{2}}\right)_{x} \in L^{2}\left(Q_{T}\right) \quad \forall \alpha \geqslant \alpha_{0} .
\end{aligned}
$$

Case 2. Let $2<n<3,1<m<2$, and $r$ satisfy both $0<r<1$ and $0<2+r-n$. If $h_{\epsilon}$ is the unique positive smooth solution to the regularized problem (36)-(38) with

$$
\delta(\epsilon)=\epsilon^{\theta} \quad \theta<1 / 2 .
$$

Then all of the above results hold, and $h$ has the further regularity

$$
\begin{aligned}
& h^{1+r / 2} \in L^{2}\left(0, T ; H^{2}\left(S^{\mathrm{E}}\right)\right) \\
& \left(h^{\alpha}\right)_{x} \in L^{4}\left(Q_{T}\right) \quad \forall \alpha \geqslant \frac{r+2}{4} .
\end{aligned}
$$

We remark that there is an existence theorem for $n=2$ which is very similar to case 2 , and refer the reader to [6] for the slightly different definition of weak solution needed.

Proof. We first prove case 1 , in which $n \geqslant 3$. Recall that $h_{\epsilon}$ is smooth so that we can integrate by parts:

$\frac{\mathrm{d}}{\mathrm{d} t} \frac{1}{2} \int_{S^{1}} h_{\epsilon x}^{2}=-\int_{S^{\mathrm{I}}} f_{\epsilon}\left(h_{\epsilon}\right) h_{\epsilon x x x}^{2}-\frac{m(m-1)(2-m)}{3} \int_{S^{1}} h_{\epsilon}{ }^{m-3} h_{\epsilon x}^{4}-m \int_{S^{1}} h_{\epsilon}{ }^{m-1} h_{\epsilon x x}^{2}$.

Note that all the terms on the right-hand side have the same sign if $1<m<2$. Integrating in time gives

$$
\begin{aligned}
\frac{1}{2} \int_{S^{1}} h_{\epsilon x}^{2}(\cdot, 0) & =\frac{1}{2} \int_{S^{!}} h_{\epsilon x}^{2}(\cdot, T)+\iint_{Q_{T}} f_{\epsilon}\left(h_{\epsilon}\right) h_{\epsilon x x x}^{2}+\frac{m(m-1)(2-m)}{3} \iint_{Q_{T}} h_{\epsilon}{ }^{m-3} h_{\epsilon x}^{4} \\
+ & m \iint_{Q_{T}} h_{\epsilon}^{m-1} h_{\epsilon x x}^{2}
\end{aligned}
$$

which then guarantees a priori bounds independent of $\epsilon$ for

$$
\begin{gathered}
\int_{\mathcal{S}^{1}} h_{\epsilon_{x}}^{2}(\cdot, t) \quad \iint_{Q_{T}} f_{\epsilon}\left(h_{\epsilon}\right) h_{\epsilon x x x}^{2}, \iint_{Q_{T}} h_{\epsilon}{ }^{m-3} h_{\epsilon_{x}}^{4}=c_{0} \iint_{Q_{T}}\left(h_{\epsilon}^{\frac{m+1}{4}}\right)_{x} \\
\text { and } \iint_{Q_{T}} h_{\epsilon}{ }^{m-1} h_{\epsilon x x}^{2}
\end{gathered}
$$

in terms of the initial data, $\int_{S^{1}} h_{\epsilon x}^{2}(\cdot, 0)$.

Note that for any $h_{\epsilon}$ smooth and bounded away from zero:

$$
\begin{aligned}
\int_{S^{1}}\left(h_{\epsilon}{ }^{\frac{m+1}{2}}\right)_{x x}^{2} & =\left(\frac{m+1}{2}\right)^{2}\left[\int_{S^{1}} h_{\epsilon}^{m-1} h_{\epsilon x x}^{2}\right. \\
& \left.+\left((m-1)^{2}+\frac{(m-1)(m-2)}{3}\right) \int_{S^{1}} h_{\epsilon}{ }^{m-3} h_{\epsilon_{x}}^{4}\right] .
\end{aligned}
$$


Therefore $\int\left(h_{\epsilon}^{\frac{m+1}{2}}\right)_{x x}^{2}$ is a linear combination of a priori bounded quantities, hence is $a$ priori bounded. Thus the following are bounded uniformly in $\epsilon$ :

$$
\iint_{Q_{T}}\left(h_{\epsilon}^{\frac{m+1}{2}}\right)_{x x}^{2} \quad \iint_{Q_{T}}\left(h_{\epsilon}^{\frac{m+1}{4}}\right)_{x^{*}}^{4}
$$

We obtain further a priori bounds by noting that integration by parts also implies

$$
\begin{aligned}
\frac{\mathrm{d}}{\mathrm{d} t} \int_{S^{1}} h_{\epsilon}^{\alpha}= & -\alpha(\alpha-1)\left[\int_{S^{1}} h_{\epsilon}^{\alpha-2} f_{\epsilon}\left(h_{\epsilon}\right) h_{\epsilon x x}^{2}-\frac{1}{3} \int_{S^{1}}\left(f_{\epsilon}\left(h_{\epsilon}\right) h_{\epsilon}^{\alpha-2}\right)^{\prime \prime} h_{\epsilon x}^{4}\right. \\
& \left.+m \int_{S^{1}} h_{\epsilon}^{\alpha-3+m} h_{\epsilon_{x}}^{2}\right] .
\end{aligned}
$$

Integrating in time implies.

$$
\begin{gathered}
m \iint_{Q_{T}} h_{\epsilon}^{\alpha-3+m} h_{\epsilon x}^{2}=-\iint_{Q_{T}} h_{\epsilon}^{\alpha-2} f_{\epsilon}\left(h_{\epsilon}\right) h_{\epsilon x x}^{2}+\frac{1}{3} \iint_{Q_{T}}\left(f_{\epsilon}\left(h_{\epsilon}\right) h_{\epsilon}^{\alpha-2}\right)^{\prime \prime} h_{\epsilon x}^{4} \\
+\frac{1}{\alpha(1-\alpha)}\left(\int_{S^{1}} h_{\epsilon}^{\alpha}(\cdot, T)-\int_{S^{1}} h_{\epsilon}^{\alpha}(\cdot, 0)\right) \\
\leqslant\left|h_{\epsilon}{ }^{\alpha-1-m} f_{\epsilon}\left(h_{\epsilon}\right)\right|_{L^{\infty}} \int_{S^{1}} h_{\epsilon}^{m-1} h_{\epsilon x x}^{2}+\frac{1}{3}\left|\left(f_{\epsilon}\left(h_{\epsilon}\right) h_{\epsilon}^{\alpha-2}\right)^{\prime \prime} h_{\epsilon}{ }^{3-m}\right|_{L^{\infty}} \int_{S^{1}} h_{\epsilon}{ }^{m-3} h_{\epsilon x}^{4} \\
+\frac{1}{\alpha|1-\alpha|}\left(\int_{S^{1}} h_{\epsilon}^{\alpha}(\cdot, T)+\int_{S^{1}} h_{\epsilon}^{\alpha}(\cdot, 0)\right) .
\end{gathered}
$$

Note that, since $\alpha-1+n>m$, a variant of an argument in the appendix of [6] implies that $\left|h_{\epsilon}{ }^{\alpha-1-m} f_{\epsilon}\left(h_{\epsilon}\right)\right|_{L^{\infty}}$ and $\left|\left(f_{\epsilon}\left(h_{\epsilon}\right) h_{\epsilon}{ }^{\alpha-2}\right)^{\prime \prime} h_{\epsilon}{ }^{3-m}\right|_{L^{\infty}}$ are $a$ priori bounded. Furthermore, since $\left|h_{\epsilon}(x, t)\right| \leqslant M$ for all $x$ and $t, \int h_{\epsilon}{ }^{\alpha}(\cdot, T)$ and $\int h_{\epsilon}{ }^{\alpha}(\cdot, 0)$ are bounded. These facts and (43) yield an $a$ priori bound for $\int_{Q T} h_{\epsilon}^{\alpha-3+m} h_{\epsilon x}^{2}$.

By weak compactness, these bounds imply that there exists a subsequence so that

$$
\begin{aligned}
& h_{\epsilon}^{\frac{m+1}{2}} \rightarrow h^{\frac{m+1}{2}} \quad \text { in } L^{2}\left(0, T ; H^{2}\left(S^{1}\right)\right) \\
& \left(h_{\epsilon}{ }^{\gamma}\right)_{x} \rightarrow\left(h^{\gamma}\right)_{x} \quad \text { in } L^{4}\left(0, T ; L^{4}\left(S^{1}\right)\right) \quad \forall y \geqslant \frac{m+1}{4}- \\
& \left(h_{\epsilon}{ }^{\frac{\alpha+m-1}{2}}\right)_{x} \rightarrow\left(h^{\frac{\alpha+m-1}{2}}\right)_{x} \quad \text { in } L^{2}\left(0, T ; L^{2}\left(S^{1}\right)\right) \quad \forall \alpha \geqslant \alpha_{0} \\
& h_{\epsilon x} \rightarrow h_{x} \quad \text { in } L^{\infty}\left(0, T ; L^{2}\left(S^{1}\right)\right) .
\end{aligned}
$$

Given a test function $\varphi \in C_{0}^{\infty}\left(0, T ; C^{\infty}\left(S^{1}\right)\right)$, the approximate solution $h_{\epsilon}$ satisfies the integral equation

$$
\begin{aligned}
\iint_{Q_{T}} h_{\epsilon} \varphi_{t}+ & \frac{3}{2} \iint_{Q_{T}} f_{\epsilon}^{\prime}\left(h_{\epsilon}\right) h_{x}^{2} \varphi_{x x}+\frac{1}{2} \iint_{Q_{T}} f_{\epsilon}^{\prime \prime}\left(h_{\epsilon}\right) h_{\epsilon}^{3} \varphi_{x}+\iint_{Q_{T}} f_{\epsilon}\left(h_{\epsilon}\right) h_{\epsilon x} \varphi_{x x x} \\
& =\iint_{Q_{T}}\left(h_{\epsilon}{ }^{m}\right)_{x} \varphi_{x} .
\end{aligned}
$$

To prove that the weak limit $h$ satisfies (39), we must show convergence of the nonlinear terms in (48). We present the argument for $\int_{Q_{T}} f_{\epsilon}^{\prime \prime}\left(h_{\epsilon}\right) h_{\epsilon_{x}}^{3} \varphi_{x}$. The other terms follow analogously.

Lemma 4.2. Let $\Omega \subset \subset Q_{T}$ be compactly contained in $Q_{T}$. Then

$$
f_{\epsilon}^{\prime \prime}\left(h_{\epsilon}\right) h_{\epsilon_{x}}^{2} \rightarrow f^{\prime \prime}(h) h_{x}^{2} \text { strongly in } L^{2}(\Omega) \text {. }
$$


Proof. This is identical to the proof in [6] for the lubrication approximation. Fix $\mu>0$.

$$
\begin{gathered}
\iint_{\Omega}\left(f_{\epsilon}^{\prime \prime}\left(h_{\epsilon}\right) h_{\epsilon_{x}}^{2}-f^{\prime \prime}(h) h_{x}^{2}\right)^{2}=\iint_{\Omega \cap\{h \geqslant \mu\}}\left(f_{\epsilon}^{\prime \prime}\left(h_{\epsilon}\right) h_{\epsilon_{x}}^{2}-f^{\prime \prime}(h) \dot{h_{x}^{2}}\right)^{2} \\
+\iint_{\Omega \cap(h<\mu\}}\left(f_{\epsilon}^{\prime \prime}\left(h_{\epsilon}\right) h_{\epsilon_{x}}^{2}-f^{\prime \prime}(h) h_{x}^{2}\right)^{2} .
\end{gathered}
$$

By the regularity theory of uniformly parabolic equations, $h$ is smooth in $\Omega \cap\{h \geqslant \mu\}$, hence $h_{\epsilon}$ and its derivatives converge uniformly to $h$ and its derivatives on this set. Taking $\epsilon$ to zero,

$$
\iint_{\Omega \cap(h \geqslant \mu\}}\left(f_{\epsilon}^{\prime \prime}\left(h_{\epsilon}\right) h_{\epsilon x}^{2}-f^{\prime \prime}(h) h_{x}^{2}\right)^{2} \rightarrow 0 .
$$

For the second integral, we expand the square and bound each term. Taking $\gamma=\frac{m+1}{4}$, we find that one term is

$$
\begin{gathered}
\iint_{\Omega \cap\{h<\mu\}}\left(f_{\epsilon}^{\prime \prime}\left(h_{\epsilon}\right)\right)^{2} h_{\epsilon x}^{4} \leqslant C \iint_{h<\mu} h_{\epsilon}^{4-4 y}\left(f_{\epsilon}^{\prime \prime}\left(h_{\epsilon}\right)\right)^{2}\left(h_{\epsilon}{ }^{\gamma}\right)_{x}^{4} \\
\leqslant C\left(\sup _{\{h<\mu\}} h_{\epsilon}^{4-4 \gamma}\left(f_{\epsilon}^{\prime \prime}\left(h_{\epsilon}\right)\right)^{2}\right) \iint_{Q_{T}}\left(h_{\epsilon}{ }^{\gamma}\right)_{x}^{4} \\
\leqslant C \sup _{\{h<\mu\}} h_{\epsilon}^{4-4 \gamma}\left(f_{\epsilon}^{\prime \prime}\left(h_{\epsilon}\right)\right)^{2} .
\end{gathered}
$$

In the above we used the fact that $\left(h_{\epsilon}{ }^{\gamma}\right)_{x}$ is uniformly bounded in $L^{4}\left(Q_{T}\right)$. We now use the fact that $f_{\epsilon}^{\prime \prime}(y) \rightarrow f^{\prime \prime}(y)$ uniformly on $[0, M]$ as $\epsilon \rightarrow 0$ for $n>2$, as proved in the appendix of [6]. This and the uniform convergence of $h_{\epsilon}$ to $h$ imply $f_{\epsilon}^{\prime \prime}\left(h_{\epsilon}\right)$ converges uniformly on $Q_{T}$ to $f^{\prime \prime}(h)$. Therefore, by taking $\epsilon$ small,

$$
\sup _{\{h<\mu\}} h_{\epsilon}^{4-4 \gamma}\left(f_{\epsilon}^{\prime \prime}\left(h_{\epsilon}\right)\right)^{2} \leqslant C \mu^{4-4 \gamma+2(n-2)} \text {. }
$$

Recall that $\frac{m+1}{4}=\gamma<\frac{n}{2}$, implying that $4-4 \gamma+2(n-2)>0$. The other two terms from the integral over $\Omega \cap\{h<\mu\}$ are bounded in the same way. Taking $\mu \rightarrow 0$, we have the result.

This Iemma implies

$$
\iint_{Q_{T}} f_{\epsilon}^{\prime \prime}\left(h_{\epsilon}\right) h_{\epsilon x}^{3} \varphi_{x} \rightarrow \iint_{Q_{T}} f^{\prime \prime}(h) h_{x}^{3} \varphi_{x}
$$

since $\varphi$ has support $\Omega$ compact in $Q_{T}$ and $h_{\epsilon x}$ converges weakly to $h_{x}$ in $L^{2}\left(Q_{T}\right)$. The other nonlinear terms converge similarily. This finishes the proof for the case $n \geqslant 3$.

We now prove case 2 , in which $2<n<3$. This uses a convex entropy first introduced in [6]. Take $G_{n \epsilon}^{r}(y)$ so that $G_{n \epsilon}^{r^{\prime \prime}}(y)=\frac{y^{r}}{f_{\epsilon}(y)}$ for $y>0$.

$$
G_{n \epsilon}^{r}(y)=\frac{y^{r-n+2}}{(2-n+r)(1-n+r)}+c+\frac{\epsilon y^{r-2}}{(r-2)(r-3)}
$$

where $c$ is chosen so that $\int_{S^{1}} G_{n \epsilon}^{r}\left(h_{\epsilon}\right) \geqslant 0$.

As before,

$\frac{\mathrm{d}}{\mathrm{d} t} \int_{S^{1}} G_{n \epsilon}^{r}\left(h_{\epsilon}\right)=-\int_{S^{1}} h_{\epsilon}{ }^{r} h_{\epsilon x x}^{2}-\frac{1}{3} r(1-r) \int_{S^{1}} h_{\epsilon}{ }^{r-2} h_{\epsilon x}^{4}-m \int_{S^{1}} \frac{h_{\epsilon}{ }^{r+m-1}}{f_{\epsilon}\left(h_{\epsilon}\right)} h_{\epsilon_{x}}^{2}$ 
We note that all the terms on the right-hand side have the same sign since $0<r<1$. Integrating in time gives

$$
\begin{gathered}
\int_{S^{1}} G_{n \epsilon}^{r}\left(h_{\epsilon}(\cdot, T)\right)+\iint_{Q_{T}} h_{\epsilon}{ }^{r} h_{\epsilon x x}^{2}+\frac{1}{3} r(1-r) \iint_{Q_{T}} h_{\epsilon}^{r-2} h_{\epsilon}^{4}+m \iint_{Q_{T}} \frac{h_{\epsilon}{ }^{r+m-1}}{f_{\epsilon}\left(h_{\epsilon}\right)} h_{\epsilon x}^{2} \\
=\int_{S^{1}} G_{n \epsilon}^{r}\left(h_{\epsilon}(\cdot, 0)\right) .
\end{gathered}
$$

The constraint (40) on $\delta(\epsilon)$ and $r-n+2>0$ provide an a priori bound for the entropy of the initial data, $\int_{S^{1}} G_{n \epsilon}^{r}\left(h_{\epsilon}(\cdot, 0)\right) \leqslant C$, where $C$ is independent of $\epsilon$. In this way, the following are bounded independent of $\epsilon$

$$
\begin{gathered}
\iint_{Q_{T}} h_{\epsilon}{ }^{r} h_{\xi_{x x}}^{2}, \iint_{Q_{T}} h_{\epsilon}^{r-2} h_{\epsilon_{x}}^{4}=c_{1} \iint_{Q_{T}}\left(h_{\epsilon}^{\frac{r}{4}+\frac{1}{2}}\right)_{x}^{4}, \iint_{Q_{T}} h_{\epsilon}^{r+m-n-1} h_{\epsilon_{x}}^{2} \\
=c_{2} \iint_{Q_{T}}\left(h_{\epsilon}^{\frac{r+m-n+1}{2}}\right)_{x^{*}}^{2}
\end{gathered}
$$

The above used the fact that there exists $C$ independent of $\epsilon$ such that $f_{\epsilon}\left(h_{\epsilon}\right) \leqslant C h_{\epsilon}{ }^{n}$.

For a smooth function bounded away from zero,

$\int_{S^{t}}\left(h_{\epsilon}{ }^{1+r / 2}\right)_{x x}^{2}=(1+r / 2)^{2}\left(\int_{S^{1}} h_{\epsilon}^{r} h_{\epsilon x x}^{2}+\left\{\frac{r^{2}}{4}-\frac{r}{3}(r-1)\right\} \int_{S^{1}} h_{\epsilon}^{r-1} h_{\epsilon x}^{4}\right)$.

As before, this shows that $\int\left(h_{\epsilon}{ }^{1+r / 2}\right)_{x x}^{2}$ is a linear combination of a priori bounded quantities, hence is a priori bounded. These bounds imply that for fixed $r$, there exists a subsequence so that in addition to the weak convergences (44), (45) and (47) we have

$$
\begin{aligned}
& \left(h_{\epsilon}{ }^{1+\frac{r}{2}}\right)_{x x} \rightarrow\left(h^{1+\frac{r}{2}}\right)_{x x} \quad \text { in } L^{2}\left(0, T ; L^{2}\left(S^{1}\right)\right), \\
& \left(h_{\epsilon}^{\alpha}\right)_{x} \rightarrow\left(h^{\alpha}\right)_{x} \quad \text { in } L^{4}\left(0, T ; L^{4}\left(S^{\mathrm{l}}\right)\right) \quad \forall \alpha \geqslant \frac{r}{4}+\frac{1}{2} \\
& \left(h_{\epsilon}^{\frac{c+m+l=n}{2}}\right)_{x} \rightarrow\left(h^{\frac{r+m+l=n}{2}}\right)_{x} \quad \text { in } L^{2}\left(0, T ; L^{2}\left(S^{1}\right)\right) .
\end{aligned}
$$

We note that the proof of the bound needed for the weak convergence (46) uses the uniform bounds on $\left.\iint h_{\epsilon}{ }^{r-2} h_{\epsilon}\right)_{x}^{4}$ and $\iint h_{\epsilon}{ }^{r} h_{\epsilon x x}^{2}$.

The rest of the proof follows as in the case $n \geqslant 3$, in that these weak convergences imply that the nonlinear terms converge. For example, $(51)$ is sufficient to prove lemma 4.2. The reader is also referred to [6] for a discussion.

Proposition 4.3. Given $1<n<2,1<m<2, h_{0} \geqslant 0$, and $h_{0} \in H^{1}\left(S^{1}\right)$. Let $h_{\epsilon}$ be the unique positive smooth solution to the regularized problem $(36)-(38)$ with

$$
\delta(\epsilon)=\epsilon^{\theta} \quad \theta<\frac{2}{5} .
$$

Then on any time interval $[0, T]$, there exists a subsequence of $\left\{h_{\epsilon}\right\}$ that converges pointwise uniformly and weakly in

$$
L^{2}\left(0, T ; H^{2}\left(S^{1}\right)\right) \cap L^{\infty}\left(0, T ; H^{1}\left(S^{1}\right)\right)
$$

to a solution $h$ in the sense of distributions

$$
\iint_{Q_{T}} h \varphi_{t}=\iint_{Q_{T}} f(h) h_{x x} \varphi_{x x}+\iint_{Q_{T}} f^{\prime}(h) h_{x} h_{x x} \varphi_{x}+\iint_{Q_{T}}\left(h^{m}\right)_{x} \varphi_{x} .
$$


Furthermore, given any $0<s<\min \left(2-n, \frac{1}{2}\right)$ and any $\alpha_{0}>\max (0,3 / 2-n)$, there exists a solution with the additional regularity:

$$
\begin{aligned}
& h^{1-\frac{s}{2}}, h^{\frac{m+1}{2}} \in L^{2}\left(0, T ; H^{2}\left(S^{1}\right)\right) \\
& \left(h^{\frac{\alpha-1+m}{2}}\right)_{x} \in L^{2}\left(Q_{T}\right) \quad \forall \alpha \geqslant \alpha_{0} \\
& \left(h^{\gamma}\right)_{x} \in L^{4}\left(Q_{T}\right) \quad \forall \gamma \geqslant \min \left(\frac{1}{2}-\frac{s}{4}, \frac{m+1}{4}\right) \\
& h^{\frac{1-S+m-n}{2}} \in L^{2}\left(0, T ; H^{\mathrm{t}}\left(S^{\mathrm{l}}\right)\right) .
\end{aligned}
$$

We remark that one can prove an existence theorem for $3 / 8<n \leqslant 1$ which is very similar to the above theorem, and refer the reader to [6] for the definition of weak solution needed. There is also an existence theorem for $0<n \leqslant 3 / 8$ which uses a weaker definition of weak solution than a distribution solution. Again, the interested reader is referred to [6] for the details.

Proof. The following proof is almost identical to the existence proof in [6] for the equation (7) without the 'porous media' term for $1<n<2$. The main difference is the higher regularity (58) obtained from the presence of the 'porous media' term in the $G_{s}$ entropy dissipation. For this reason, we only sketch the proof.

Recall the convex entropy $G_{0}^{\prime \prime}(y)=\frac{1}{f(y)}$ introduced in section 3 . Integration by parts yields (35):

$$
\int_{S^{1}} \frac{\epsilon}{6 h_{\epsilon}(x, t)^{2}} \mathrm{~d} x+\int_{S^{1}} G_{0}\left(h_{\epsilon}(x, t)\right) \mathrm{d} x+\int_{0}^{t} \int_{S^{1}} h_{\epsilon_{x x}}^{2}+m \int_{0}^{t} \int_{S^{1}} \frac{h_{\epsilon}{ }^{m-1}}{f_{\epsilon}\left(h_{\epsilon}\right)} h_{\epsilon_{x}}^{2} \leqslant C_{\epsilon} .
$$

The constraint (53) on $\delta(\epsilon)$ and $n<2$ provide an a priori bound for the entropy of the initial data $C_{\epsilon}=\int G_{0}\left(h_{\epsilon 0}\right)+\int \epsilon /\left(6 h_{\epsilon 0}^{2}\right) \leqslant C$, where $C$ is independent of $\epsilon$. Mass conservation and the convexity of $G_{0}$ allow us to choose $G_{0}$ so that $\int G_{0}\left(h_{\epsilon}\right) \geqslant 0$. Therefore

$$
\iint_{Q T} h_{\epsilon x x}^{2} \leqslant C
$$

Weak compactness implies that there exists a subsequence that converges weakly in $L^{2}\left(0, T ; H^{2}\left(S^{1}\right)\right)$ to $h$. Dissipation of surface tension energy (22) implies that $\partial h_{\epsilon} / \partial t$ is uniformly bounded in $L^{2}\left(0, T ; H^{-1}\left(S^{1}\right)\right)$. The well-known Lions-Aubin lemma [23] then implies that there exists a subsequence that converges strongly in $L^{2}\left(0, T ; H^{\mathrm{l}}\left(S^{\mathrm{l}}\right)\right)$ to $h$.

We now prove that $h$ is a weak solution in the sense (54). Since $h_{\epsilon}$ converges pointwise uniformly to $h$ on $Q_{T}$ and $f_{\epsilon}^{\prime}(y)$ converges uniformly to $f^{\prime}(y)$ on $[0, M]$ for $n>1$ (as proved in the appendix of [6]), $f_{\epsilon}^{\prime}\left(h_{\epsilon}\right)$ converges uniformly to $f^{\prime}(h)$ on $Q_{T}$. Similarly $f_{\epsilon}\left(h_{\epsilon}\right)$ converges uniformly to $f(h)$ on $Q_{T}$. Hence the limit $h(x, t)$ solves the equation in the sense (54). For example,

$$
\iint_{Q_{T}} f_{\epsilon}^{\prime}\left(h_{\epsilon}\right) h_{\epsilon x} h_{\epsilon x x} \varphi_{x} \rightarrow \iint_{Q_{T}} f^{\prime}(h) h_{x} h_{x x} \varphi_{x}
$$

since $h_{\epsilon x x}$ converges weakly in $L^{2}$ to $h_{x x}, h_{\epsilon x}$ converges strongly in $L^{2}$ to $h_{x}$, and $f_{\epsilon}^{\prime}\left(h_{\epsilon}\right)$ converges uniformly to $f^{\prime}(h)$.

For the extra regularity, we recall a convex entropy first introduced in [6]

$$
G_{n \epsilon}^{-s}(y)=\frac{1}{(2+s)(3+s)} \frac{\epsilon}{y^{2+s}}+\frac{1}{(2-n-s)(1-n-s)} y^{2-n-s}+c
$$


chosen so that $\left(G_{n \epsilon}^{-s}\right)^{\prime \prime}(y)=\frac{1}{y^{s} f_{\epsilon}(y)}$. By integration by parts,

$\frac{\mathrm{d}}{\mathrm{d} t} \int_{S^{1}} G_{n \epsilon}^{-s}\left(h_{\epsilon}\right)=-\int_{\mathcal{S}^{1}} h_{\epsilon}^{-s} h_{\epsilon x x}^{2}+\frac{s(s+1)}{3} \int_{S^{1}} h_{\epsilon}^{-s-2} h_{\epsilon x}^{4}-m \int_{S^{1}} \frac{h_{\epsilon}^{m-1-s}}{f_{\epsilon}\left(h_{\epsilon}\right)} h_{\epsilon_{x}}^{2}$.

We note that one of the coefficients, $\frac{s(s+1)}{3}$, is positive, hence the techniques used above do not immediately apply. However, it is possible to 'hide' the $\iint h_{\epsilon}{ }^{-s-2} h_{\epsilon x}^{4}$ term in the $\iint h_{\epsilon}{ }^{-s} h_{\epsilon x x}^{2}$ term in such a way as to bound the right-hand side from above by a combination of the integrals where all the coefficients are negative. This requires $s<\frac{1}{2}$, and the details are fully presented in [6]. As before, we then have a priori upper bounds for the following:

$$
\iint_{Q T}\left(h_{\epsilon}^{1 / 2-s / 4}\right)_{x}^{4} \quad \iint_{Q \tau}\left(h_{\epsilon}{ }^{1-s / 2}\right)_{x x}^{2}
$$

This used $s<2-n$ and the constraint (53) on $\delta(\epsilon)$. Again, energy dissipation yields $a$ priori bounds for

$$
\iint_{Q_{T}}\left(h_{\epsilon}^{\frac{\pi+1}{4}}\right)_{x}^{4} \quad \iint_{Q_{T}}\left(h_{\epsilon}^{\frac{m+1}{2}}\right)_{x x}^{2}
$$

The uniform bound. for $\int h_{\epsilon}^{\alpha-3+n} h_{\epsilon x}^{2}$ follows as before, and uses the bounds on $\iint h_{\epsilon}{ }^{-s-2} h_{\epsilon x}^{4}$ and $\iint h_{\epsilon}{ }^{-s} h_{\epsilon x x}^{2}$. This requires $\alpha_{0}>3 / 2-n$.

Taking a subsequence of the above subsequence, the limit $h$ inherits these bounds, hence has the desired regularity.

\section{Long time behaviour of solutions}

In this section we prove the long time results for the equations.

Proposition 5.1. Given $h_{0} \in H^{1}\left(S^{1}\right), h_{0} \geqslant 0$, let $h$ be the weak solution from section 4. The mean of the initial data is denoted $\bar{h}, \bar{h}=\frac{1}{\left|S^{1}\right|} \int_{S^{1}} h_{0}$.

Case 1. $n \geqslant 3$. There exist positive $A$ and $c$ such that for all $t \in[0, T]$

$$
\|h(\cdot, t)-\bar{h}\|_{L^{\infty}} \leqslant A \mathrm{e}^{-c t} \text {. }
$$

$A$ is determined by $\left|h_{0}\right|_{H^{1}}$ and $\left|S^{1}\right|$. The rate of decay, $c$, is determined by $\left|S^{1}\right|$ and $\bar{h}$. In particular, if $h_{0}$ is nonzero the solution is a positive strong solution after a critical time $T^{*}$.

Case 2. $0<n<3$. There exist positive $A$ and $c$ such that for all $t \in[0, T]$,

$$
\|h(x, t)-\bar{h}\|_{L^{\infty}} \leqslant A \mathrm{e}^{-c t} \text {. }
$$

$A$ is determined by $\left|h_{0}\right|_{H^{1}}, n, \bar{h}$, and $\left|S^{1}\right|$. The rate of decay, $c$, is determined by $n$ and $\vec{h}$. In particular, if $h_{0}$ is nonzero there exists a time $T^{*}$, after which the solution is a positive strong solution.

Proof. We first prove case $1, n \geqslant 3$. We recall the energy dissipation (41):

$$
\frac{\mathrm{d}}{\mathrm{d} t} \frac{1}{2} \int_{S^{1}} h_{\epsilon_{x}}^{2}=-\int_{S^{1}} f_{\epsilon}\left(h_{\epsilon}\right) h_{\epsilon x x x}^{2}-\frac{m(m-1)(2-m)}{3} \int_{S^{1}} h_{\epsilon}^{m-3} h_{\epsilon x}^{4}-m \int_{S^{1}} h_{\epsilon}^{m-1} h_{\epsilon x x^{*}}^{2}
$$

The terms on the right-hand side are all nonpositive, hence

$$
\frac{\mathrm{d}}{\mathrm{d} t} \frac{1}{2} \int_{S^{1}} h_{\epsilon_{x}}^{2} \leqslant-\frac{m(m-1)(2-m)}{3} \int_{S^{1}} h_{\epsilon}^{m-3} h_{\epsilon_{x}}^{4} \leqslant-C \int_{S^{1}} h_{\epsilon_{x}}^{4}
$$


In the above, we used the fact that $\left\|h_{\epsilon}(\cdot, t)\right\|_{\infty} \leqslant M$ for all $t \in[0, T]$, hence $h_{\epsilon}(x, t)^{m-3} \geqslant C$. Schwarz's inequality then implies

$$
\frac{\mathrm{d}}{\mathrm{d} t} \int_{S^{1}} h_{\epsilon_{x}}^{2} \leqslant-C\left(\int_{S^{1}} h_{\epsilon_{x}}^{2}\right)^{2} .
$$

A variant of Gronwall's lemma gives

$$
\int_{S^{1}} h_{\epsilon x}^{2}(x, t) \mathrm{d} x \leqslant \frac{\int_{S^{1}} h_{\epsilon_{x}}^{2}(x, 0) \mathrm{d} x}{1+C t \int_{S^{1}} h_{\epsilon_{x}}^{2}(x, 0) \mathrm{d} x}<\frac{1}{C t} .
$$

We note that $C$ depends on $\left|h_{0}\right|_{H^{1}}$, through its dependence on $\left|h_{0}\right|_{\infty}$. The weak convergence $h_{\epsilon x} \rightarrow h_{x}$ in $L^{2}\left(S^{1}\right)$ implies that the weak limit inherits the above bound:

$$
\int_{S^{1}}(h(x, t)-\bar{h})^{2} \mathrm{~d} x \leqslant A \int_{S^{1}} h_{x}^{2}(x, t) \mathrm{d} x \leqslant \frac{A}{C t} .
$$

In the above we used Poincaré's inequality:

Poincaré's inequality. Let $h \in C^{2}\left(S^{1}\right)$ and consider $x_{0} \in S^{1}$. Then

$$
\int_{S^{1}}\left(h(x)-h\left(x_{0}\right)\right)^{2} \mathrm{~d} x \leqslant\left|S^{1}\right|^{2} \int_{S^{1}} h_{x}^{2} \mathrm{~d} x
$$

This also holds true for $h \in H^{1}\left(S^{1}\right)$ by a density argument.

The following interpolation inequality is proven in [6]:

$$
|w|_{L^{\infty}} \leqslant\left(\frac{1+\alpha}{\alpha}\right)^{\alpha /(1+\alpha)}|w|_{\alpha}^{1 /(1+\alpha)}|w|_{L^{1}}^{\alpha /(1+\alpha)}+\left(\frac{1+\alpha}{\alpha}\right)|w|_{L^{1}} /\left|S^{1}\right|
$$

where $|w|_{\alpha}$ is the Hölder $-\alpha$ seminorm. Taking $\alpha=\frac{1}{2}$, in the interpolation inequality gives

$$
|h(\cdot, t)-\bar{h}|_{L^{\infty}} \leqslant \frac{A}{C t^{1 / 3}} \quad \text { for all } t>0 .
$$

This implies that there exists a time, $T_{0}$, after which $h$ is strictly positive and bounded below by $\bar{h} / 2$ and hence a strong solution. The energy dissipation applied to this strong solution implies that for $t>T_{0}$,

$$
\frac{\mathrm{d}}{\mathrm{d} t} \int_{S^{1}} h_{x}^{2} \leqslant-\left(\frac{\bar{h}}{2}\right)^{n} \int_{S^{1}} h_{x x x}^{2} .
$$

Another application of the Poincare inequality, Gronwall's lemma, and the interpolation inequality yields the exponential decay.

The proofs of the other cases are identical to those in [6] for the equation with the lubrication term alone hence we omit the details. The key ideas are that for $0<n<2$, the convex entropy $\int G_{0}\left(h_{\epsilon}\right)$ is equivalent to the $L^{2}$ norm of $h_{\epsilon}(\cdot, t)-(\bar{h}+\delta(\epsilon))$. That it dissipates with a rate $\int_{S^{1}} h_{\epsilon x x}^{2}$ implies that the approximating solutions $h_{\epsilon}$ decay exponentially fast at a rate independent of $\epsilon$. For $1<n<3$, we use the dissipation of the following convex entropy

$$
\frac{\mathrm{d}}{\mathrm{d} t} \int_{S^{1}} G_{n \epsilon}^{r}\left(h_{\epsilon}\right) \leqslant-C_{r} \int_{S^{1}}\left(h_{\epsilon}{ }^{1+\frac{r}{2}}\right)_{x x}^{2} .
$$

The entropy $\int G_{n \epsilon}^{r}\left(h_{\epsilon}\right)$ is equivalent to the $L^{2}$ norm of $h_{\epsilon}(\cdot, t)^{1+\frac{r}{2}}-(\bar{h}+\delta(\epsilon))^{1+\frac{r}{2}}$. The long time result then follows. The proofs of equivalence of the entropies to squares of $L^{2}$ norms is due to a 'parabolic sandwich' argument in which we show that the entropies can be chosen so that their graphs are 'trapped' between two parabolas. 


\section{Numerical results}

Our numerical scheme is an adaptation of a code used in [7]. With the permission of the authors, we use some of the language from this source. The simulations use a conventional finite difference method. Specifically, it is an implicit two level scheme based on central differences. In addition, some of the numerical results presented here use a dynamically adaptive mesh composed of a fixed macro-grid and adaptive micro-grid needed for higher resolution of the 'contact line'. The finite difference scheme with a fixed non-uniform non-adaptive grid was used used in $[11,16,29]$ to compute solutions of the equation $h_{t}=-\left(h h_{x x x}\right)_{x}$. In these works, they compared their results to results from a finite element method and found excellent agreement.

We consider solutions on a periodic domain $[-1,1]$ that are symmetric about $x=0$. The equation preserves this symmetry. For this reason, we solve the equation on the interval $[0,1]$, discretized by the $N$ mesh points,

$$
0=x_{1}<x_{2}<\ldots<x_{N}=1 .
$$

At each computational time level the arrays $h_{i}$ and $p_{i}, i \in[1, \ldots, N]$, approximate $h(x, t)$ and $-h_{x x}(x, t)$, and $v_{j}, j \in[1, \ldots, N-1]$ approximates $h_{x x x}(x, t)$. The $h_{i}$ and $p_{i}$ values exist at the point $x_{i}$, while $v_{i}$ is the computed third derivative at the center of the interval, $\left(x_{i}+x_{i+1}\right) / 2$. The following picture depicts these associations:

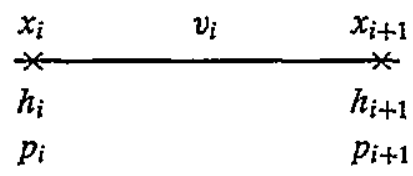

We use the notation:

$$
\begin{aligned}
& \Delta x_{i+\frac{1}{2}}=x_{i+1}-x_{i} \\
& x_{i+\frac{1}{2}}=\frac{1}{2}\left(x_{i+1}+x_{i}\right) \\
& \Delta x_{i}=x_{i+\frac{1}{2}}-x_{i-\frac{1}{2}} \\
& h_{i+\frac{1}{2}}=\frac{1}{2}\left(h_{i+1}+h_{i}\right) \\
& \partial h_{i+\frac{1}{2}}=\frac{h_{i+1}-h_{i}}{\Delta x_{i+\frac{1}{2}}} \\
& \delta^{2} h_{i}=\frac{\partial h_{i+\frac{1}{2}}-\partial h_{i-\frac{1}{2}}}{\Delta x_{i}} .
\end{aligned}
$$

For simplicity we describe the difference scheme in space first and later indicate the time step process. The equation we wish to compute has the form

$$
h_{t}+\left(f(h) h_{x x x}\right)_{x}-(P m(h))_{x x}=0 .
$$


We discretize the spatial operators by

$$
\begin{aligned}
& \left(h_{i}\right)_{t}+\frac{f\left(h_{i+\frac{1}{2}}\right) v_{i}-f\left(h_{i-\frac{1}{2}}\right) v_{i-1}}{\Delta x_{i}}-\delta^{2}\left(P m(h)_{i}\right)=0 \\
& v_{i}+\partial p_{i+\frac{1}{2}}=0 \\
& p_{i}+\delta^{2} h_{i}=0 .
\end{aligned}
$$

We impose 'periodic' boundary conditions by reflection symmetry at the endpoints.

The time discretization of the above set of differential-algebraic relations uses a simple two level scheme. In advancing from time $t$ to time $t+\mathrm{d} t$ we replace the time derivative terms by difference quotients involving the solution at the old time level (time $t$ ) and the as yet unknown solution at the new time level (time $t+\mathrm{d} t$ ). We evaluate the other terms using a weighted average of the solution at the two time levels; a typical weight is $\theta=0.55$ on the advanced time level and $1-\theta=0.45$ on the old time level:

$$
\frac{\mathrm{d} h}{\mathrm{~d} t}=N(h) \quad \text { would yield } \quad \frac{1}{\mathrm{~d} t}\left(h_{i}(t+\mathrm{d} t)-h_{i}(t)\right)=N(0.55 h(\cdot, t+\mathrm{d} t)+0.45 h(\cdot, t)) .
$$

At each time level, we have to solve a set of nonlinear equations. This is done with Newton's method. By choosing an appropriate ordering of the $3 N-1$ equations (59)-(61), the Jacobi matrix has its nonzero entries close to the diagonal. For this reason, the use of Newton's method is not a prohibitive expense.

We dynamically choose the length of the time steps to control several aspects. If the result of the time step violates any of a list of constraints, it rejects the step and tries again with a smaller step size. To avoid using unnecessarily short time steps, if we easily meet all the constraints for several steps, we increase the step size by about $20 \%$ on the next step. We now describe the constraints the scheme respects. The first constraint comes from local time truncation. Another constraint rejects any step for which the minimum of $h$ decreases by more than $10 \%$. We also require that the correction on the first iteration of Newton's method is a small fraction of the change over the step, where the initial guess at the change was the change over the previous step, corrected for any difference in $\mathrm{d} t$ 's. This allows us to solve the equations (59)-(61) in only one Newton iteration per time step, should we choose to do so.

We use graded spatial grids that are very fine near the contact line and less fine in other regions. The macro-grid is fixed for all time, and we call its intervals 'macro intervals'. At a fixed time level, each macro-interval is divided into $2^{j}$ micro intervals, where $j$ can be different for each macro-interval. The size of $h_{x x}$ and $h_{x x x}$ on the macro-interval determine whether to increase or decrease the exponent $j$ for the next time level. Specifically, if these derivatives are large, $j$ increases and if they are small, $j$ decreases. In this way, the grid is fine where high resolution is needed and coarse elsewhere.

All the simulations presented here have the $C_{0}^{\infty}\left(S^{1}\right)$ initial condition

$$
h_{0}(x)= \begin{cases}C \mathrm{e}^{-a /(x+1 / 4)^{2}} \mathrm{e}^{-a /(x-1 / 4)^{2}} & |x|<1 / 4 \\ 0 & \text { otherwise. }\end{cases}
$$

The weak solution is approximated via the regularization scheme of section 3 . That is, we fix $\epsilon$ and compute

$$
\begin{aligned}
& h_{\epsilon t}=-\left(f_{\epsilon}\left(h_{\epsilon}\right) h_{\epsilon x x x}\right)_{x}+\left(h_{\epsilon}{ }^{m}\right)_{x x} \\
& f_{\epsilon}\left(h_{\epsilon}\right)=\frac{h_{\epsilon}{ }^{4} h_{\epsilon}{ }^{n}}{\epsilon h_{\epsilon}{ }^{n}+h_{\epsilon}{ }^{4}}
\end{aligned}
$$


with initial condition

$$
h_{\epsilon 0}(x)=h_{0}(x)+\delta(\epsilon) .
$$

Theorem 3.1 guarantees that there exists a unique smooth positive solution to the regularization scheme. Propositions 4.1 and 4.3 guarantee that the regularization scheme will for suitably small $\epsilon$ produce a close approximation of the weak solution. Although no rigorous theorem exists quantifying the accuracy of the regularization, the numerics suggests that the convergence is $\mathcal{O}(\delta(\epsilon))$. We choose the spatial grids to be fine enough so that the bulk of the error in computing the weak solution comes from the epsilon regularization. That is, for fixed epsilon, we choose spatial grids to over-resolve the smooth regularized solution.

We present computation from several case studies.

\subsection{Case $1: n=3, m=3 / 2$}

For this case we choose initial data (62) with $c=1$ and $a=1 / 16$. We use $\delta(\epsilon)=\epsilon^{0.3}$ in the regularization scheme. We present calculations with three different values of $\epsilon: 10^{-9}$, $10^{-12}$, and $10^{-14}$. The corresponding values of $\delta(\epsilon)$ are $2.00 \times 10^{-3}, 2.51 \times 10^{-4}$ and $6.310 \times 10^{-5}$.

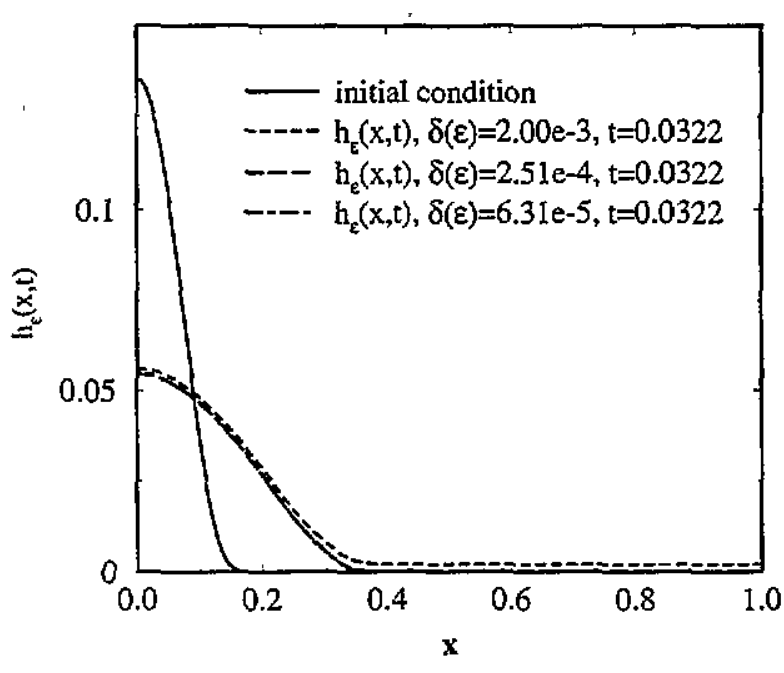

Figure 1. Approximate solutions for $n=3$, $m=3 / 2$. Pictured are solutions for three values of $\delta$ at the fixed time $t=0.0322$. Note that the 'drop' is spreading.

Figures 1-3 show the regularized solution at a fixed time $t=0.0322$ for three different values of $\epsilon$. Figures 1 and 2 depict $h_{\epsilon}(x, t)$ while figure 3 shows $h_{\epsilon x x}$. Figure 1 shows that all three values of $\epsilon$ produce approximate solutions very close to each other. Moreover they indicate that the support of the weak solution has increased from its initial support but that the support has not yet expanded to fill the entire domain.

Figure 2 shows a close up of the $h_{\epsilon}(x, t)$ near the edge of the support of weak solution. The figure clearly indicates that the convergence is $O(\delta(\epsilon))$. Moreover, it shows that the 'apparent contact line' has extremely good dependence on $\epsilon$ as $\epsilon \rightarrow 0$. Note that at the edge, the regularized solution is a monotone function. This is as expected for solutions of the porous media equation, $h_{t}=\left(h^{m}\right)_{x x}$, and should be compared to case 2 in which the fourth-order lubrication approximation dominates at the edge. 

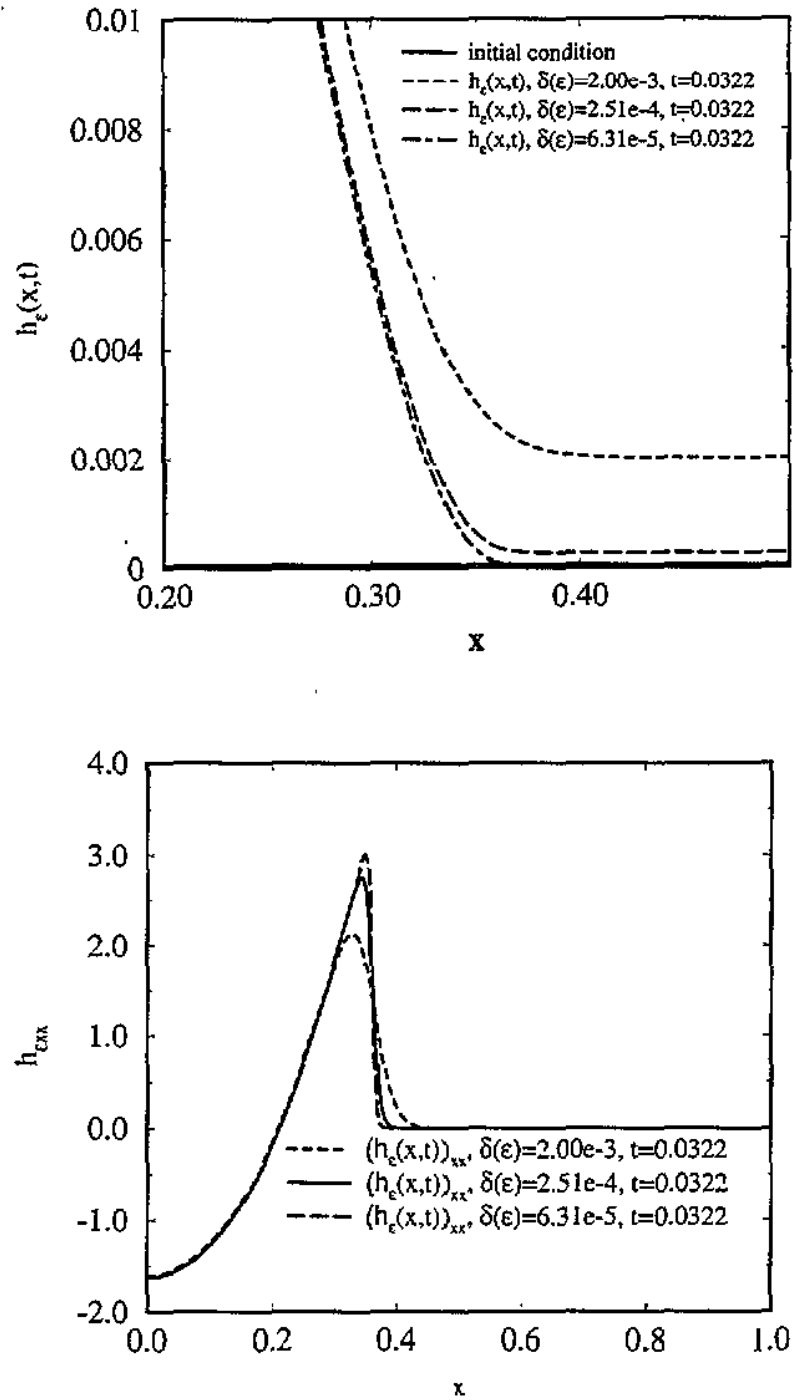

Figure 2. Close-up of approximate solutions for $n=3, m=3 / 2$. Note that the apparent 'edge' of the support has very good dependence on $\delta$ and appears to have finite speed of propagation.

Figure 3. Second derivative of approximate solutions for $n=3, m=3 / 2$. Note that the second derivatives appear to be converging to a bounded function with discontinuity 0 at the edge, consistent with the predicted quadratic touch-down.

Figure 3 shows $h_{\epsilon x x}$ for the three values of the regularization parameter. The graphs indicate that as $\epsilon \rightarrow 0$, the solution converges to a bounded function with a discontinuity at the edge of its support. Hence the weak solution appears to 'touch down' with a quadratic behaviour at its edge, as predicted by the asymptotics in section 2 for $m=3 / 2$. Hence for these values of $m$ and $n$ the fourth-order term appears to be negligible at the edge of the support.

\subsection{Case 2: $n=1$ and $m=1.9$}

For this case we also choose initial data with $c=1$ and $a=1 / 16$. We again use $\delta(\epsilon)=\epsilon^{0.3}$ in the regularization scheme. Figures $4-6$ show the regularized solution at a fixed time $t=0.0025$ for the three different values of $\epsilon: 10^{-9}, 10^{-12}$, and $10^{-14}$. Again, the corresponding values of $\delta(\epsilon)$ are $2.00 \times 10^{-3}, 2.51 \times 10^{-4}$ and $6.310 \times 10^{-5}$. Figures 
4 and 5 depict $h_{\epsilon}(x, t)$ while figure 6 shows $h_{\epsilon x x}$. As in case 1, figure 4 shows that all three values of $\epsilon$ produce approximate solutions very close to each other. Moreover they also indicate that the support of the weak solution has increased from its initial support but has not yet expanded to fill the entire domain.
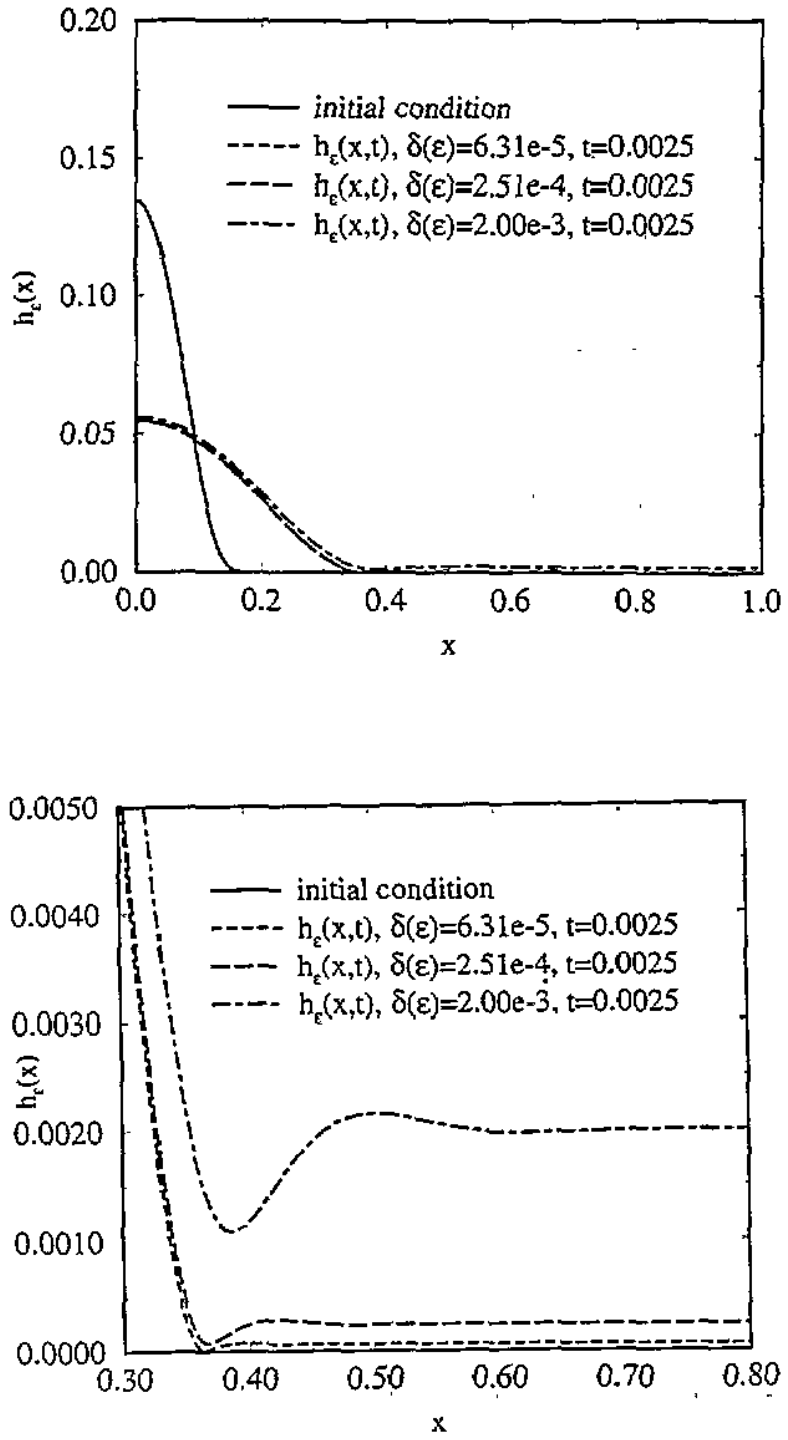

Figure 4. Approximate solutions for $n=1$. $m=1.9$. Pictured are solutions for three values of $\delta$ at the fixed time $t=0.0025$. Note that the 'drop' is spreading.
Figure 5. Close-up of approximate solutions for $n=1, m=1.9$. Note that unlike figure 2 , the approximate solution is not locally monotone at the apparent edge of the support. This is characteristic of higherorder equations.

Figure 5 shows a close up of the $h_{\epsilon}(x, t)$ near the edge of the support of weak solution. The figure clearly indicates that the convergence is $O(\delta(\epsilon))$. Moreover it shows that the edge of the support has extremely good dependence on $\epsilon$ as $\epsilon \rightarrow 0$. Note that at the edge the regularized solution is not a monotone function. That is, the height has a local minimum which is the same order of magnitude as $\delta(\epsilon)$. The solution also shows small oscillations ahead of this minimum. This 'oscillatory tail' is typical of higher order equations. This is in contrast with case 1 , in which the behaviour at the edge is determined by the lower order 'porous media' term. 


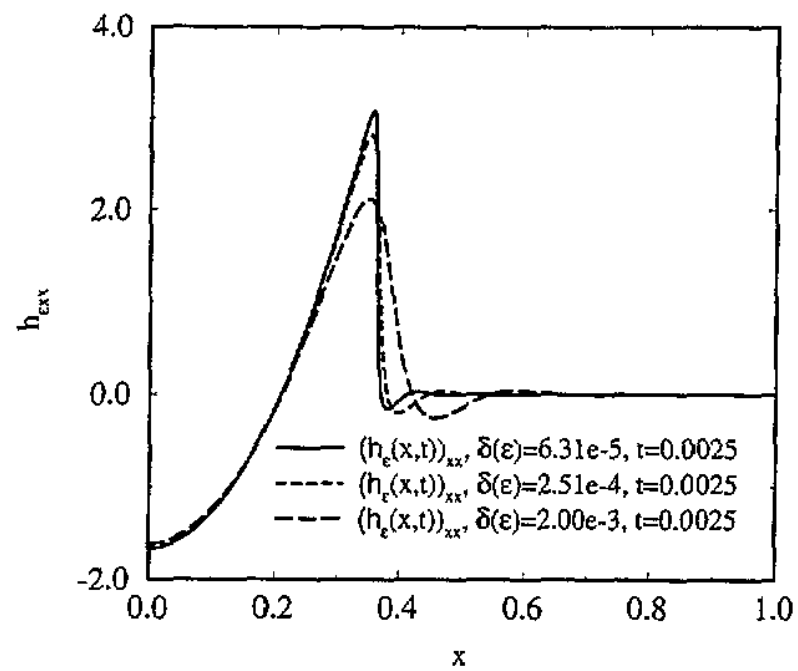

Figure 6. Second derivative of approximate solutions for $n=1, m=1.9$. Note that the second derivatives appear to be converging to a bounded function with discontinuity at the edge, consistent with the predicted quadratic touch-down.

Figure 6 shows $h_{\epsilon x x}$ for the three values of the regularization parameter. The graphs indicate that as $\epsilon \rightarrow 0$, the solution converges to a bounded function with at discontinuity at the edge of its support. Thus the weak solution appears to 'touch down' with a quadratic behaviour at its edge, which is the correct behaviour for a travelling wave solution to lubrication approximation, $h_{t}=-\left(h^{n} h_{x x x}\right)_{x}$ with $n=1$. The porous media equation with $m=1.9$ has a solution that touches down like $x^{10 / 9}$. Such behaviour is much more singular than the quadratic touchdown shown here. Hence for this case the fourth-order term dominates at the edge, as predicted by the asymptotics.

\subsection{Case 3}

Finally, we present a single calculation of a solution with the full 'van der Waals' term with a cutoff. The equation we compute is

$$
\begin{aligned}
& h_{\epsilon t}=-\left(f_{\epsilon}\left(h_{\epsilon}\right) h_{\epsilon x x x}\right)_{x}+\left(P_{m}\left(h_{\epsilon}\right)\right)_{x x} \\
& f_{\epsilon}\left(h_{\epsilon}\right)=\frac{h_{\epsilon}{ }^{4} h_{\epsilon}{ }^{3}}{\epsilon h_{\epsilon}{ }^{3}+h_{\epsilon}{ }^{4}} \\
& P_{m}\left(h_{\epsilon}\right)=\log \left[\left(\mu+h_{\epsilon}{ }^{m}\right)^{1 / m}\right] \quad m=3 / 2
\end{aligned}
$$

with initial condition

$$
h_{\epsilon 0}(x)=h_{0}(x)+\delta(\epsilon) \text {. }
$$

Note that as $\mu \rightarrow 0$ we recover the van der Waals term without a cut-off. However, for fixed $\mu>0,\left(P_{m}\left(h_{\epsilon}\right)\right)_{x}=h_{\epsilon}{ }^{m-1} h_{\epsilon x} /\left(\mu+h_{\epsilon}{ }^{m}\right)$ so that as $h_{\epsilon} \rightarrow 0$ the gradient of $P_{m}\left(h_{\epsilon}\right)$ behaves like the gradient of a 'porous media' term, $\left(h_{\epsilon}{ }^{m}\right)_{x} / m$. Note that while $\epsilon$ is a mathematical regularization of the equation, $\mu$ has the interpretation of a physical cut-off scale. Thus we fix $\mu$ small and take $\epsilon \rightarrow 0$. We remark that it is crucial that $\left(P_{m}\left(h_{\epsilon}\right)\right)_{x} / h_{\epsilon x}$ be a positive function of $h_{\epsilon}$. Otherwise this term produces an 'ill posed' backward diffusion in a range of $h_{\epsilon}$ where $\left(P_{m}\left(h_{\epsilon}\right)\right)_{x} / h_{\epsilon x}$ changes sign. It is an exercise to verify that a variant of theorem 1.2 guarantees that the regularization scheme will for suitably small $\epsilon$ produce a close approximation of the weak solution. 


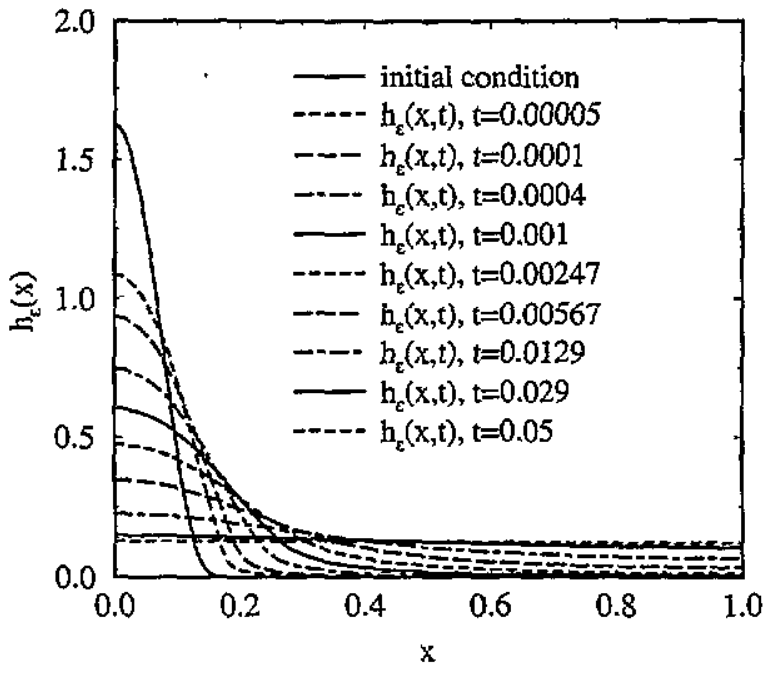

Figure 7. Approximate solutions for the problem with long-range van der Waal effects and porous media cut-off. Note that there is a pronounced 'foot' at the edge of the solution, analogous to the physical precursor film.

We choose initial data (62) with $c=12$ and $a=1$. We fix $\mu=0.00008, \epsilon=10^{-12}$, and $\delta(\epsilon)=\epsilon^{0.3}=2.51 \times 10^{-4}$. Figure 7 shows $h_{\epsilon}(x, t)$ for several times $t$. Note that the support propagates very quickly and that a 'foot' appears at the edge of the solution. This is qualitatively like the 'precursor film' [15] linked to long range van der Waals interactions in complete wetting.

\section{Conclusions}

This paper poses the equation

$$
h_{t}+\left(h^{n} h_{x x x}\right)_{x}-\left(h^{m}\right)_{x x}=0 \quad x \in S^{1}
$$

as a model problem for the study of a molecular scale cutoff of long range van der Waals forces in the moving contact line of a completely wetting thin film.

We prove a global existence theorem for weak solutions with non-negative data and consider all $n>0$ and $1<m<2$. In addition to the case where $n<3$ and sharp existence theory is known for the homogenous fourth-order equation (7), we show that with the addition of the second-order term distribution solutions exist for the critical physical case of $n=3$ (and for all $n \geqslant 3$ ) and that they become strong positive solutions in the infinite time limit.

In conjunction with the existence theory, we address leading order asymptotic analysis for the edge of the support of the solution. We examine the competition between the second and fourth-order terms and for $n>3 / 2$ this study indicates the existence theory is sharp.

We also present numerical calculations of various weak solutions. The numerics show that the solutions have support with finite speed of propagation and regularity at the edge as dictated by the asymptotics and rigorous theory.

To our knowledge none of these topics has been addressed before in the the literature for a combined second-order/fourth-order equation of this type. In fact, the sharp existence theory for the homogeneous fourth-order equation (7) with $0<n<3$ has only recently been addressed in two independent papers $[6,1]$. The question of existence of distribution solutions with increasing support for $n \geqslant 3$ is of extreme importance to the subtle physical problem of a moving contact line in a thin film, where the correct exponent is $n=3$. 
We also briefly address the full physical equations and the need for a cut-off of the singular disjoining pressure at a molecular scale. We believe that this paper is the first in either the physics or mathematics literature to address the mathematical validity of various cut-off functions of the disjoining pressure.

There are many open mathematical problems in this field. They include but are not restricted to uniqueness of a weak solution, any result in higher dimensions, questions of singularity formation (see e.g. [7, 5, 4] for a discussion). Moreover, there is a need for more comparison of the various models to actual wetting experiments.

\section{Acknowledgments}

This work was inspired by a conversation with Professor Anne Marie Cazabat from the Collège de France about possible ways to cut-off long range van der Waals interactions on a molecular scale. We are grateful to her for many enlightening conversations regarding the subtle physical problem of moving contact lines. We note that parts of the code used in the simulations were written by Todd Dupont for $[11,16]$. We thank him for useful conversations concerning the numerics. $\mathrm{AB}$ also thanks $\mathrm{M}$ Brenner for discussions concerning the physics and $\mathrm{M}$ del Pino and $\mathrm{D}$ Diller for interesting coversations about superdiffusive equations. We also thank P Constantin, L Kadanoff, and M Shelley.

Some of the computations presented in this paper were performed at the Advanced Computing Research Facility, Mathematics and Computer Science Division, Argonne National Laboratory.

Both authors are supported by NSF postdoctoral fellowships. This work was supported in part by the MRSEC Program of the National Science Foundation under Award Number DMR-9400379. This work was also supported in part by the DOE and NSF grant \# DMS9305996.

\section{References}

[1] Beretta E, Berstch M, and Dal Passo R 1994 Nonnegative solutions of a fourth-order nonlinear degenerate parabolic equation

[2] Bernis F and Friedman A 1990 Higher order nonlinear degenerate parabolic equations J. Diff. Eq. 83 179-206

[3] Bernis F, Peletier L A and Williams S M 1992 Source type solutions of a fourth-order nonlinear degenerate parabolic equation Nonlin. Anal. Theor., Methods Appl. 18(3)

[4] Bertozzi A 1994 Symmetric singularity formation in lubrication-type equations for interface motion, submitted for publication

[5] Bertozzi A L 1994 Loss and gain of regularity in a lubrication equation for thin viscous films. Proc. Int. Coll. Free Boundary Problems, Toledo, Spain, June 1993 (London: Longman) in press

[6] Bertozzi A L and Pugh M 1994 The lubrication approximation for thin viscous films: regularity and long time behaviour of weak solutions, submitted for publication

[7] Bertozzi A L, Brenner M P, Dupont T F and Kadanoff L P 1994 Singularities and similarities in interface flows Trends and Perspectives in Applied Mathematics ed L Sirovich Appl. Math. Sci. 100 (Berlin: Springer) pp 155-208

[8] Boatto S, Kadanoff L and Olla P 1993 Travelling wave solutions to thin film equations $\dot{P}$ hys. Rev. E 484423

[9] Brenner M and Bertozzi A 1993 Spreading of droplets on a solid surface Phys. Rev. Lett. 71(4) 593-6

[10] Burelbach J P, Bankoff S G and Davis S H 1988 Nonlinear stability of evaporating/condensing liquid films J. Fluid Mech. 195 463-94

[11] Constantin P, Dupont T F, Goldstein R E, Kadanoff L P, Shelley M J and Su-Min Zhou 1993 Droplet breakup in a model of the Hele-Shaw cell. Phys. Rev. E 47(6) 4169-81

[12] Daskalopoulos P and del Pino M A 1993 On fast diffusion nonlinear heat equtaions and a related singular elliptic problem Indiana Univ. Math.J. in press 
[13] Davis S H, Dibendetto E and Diller D J 1994 Some a-priori estimates for a singular evolution equation arising in thin film dynamics

[14] de Gennes P G, Hua X and Levinson P 1990 Dynamics of wetting: local contact angles J. Flutd Mech. 212 $55-63$

[15] de Gennes P E 1985 Wetting: Statics and dynamics Rev. Mod. Phys. 57

[16] Dupont T F, Goldstein R E, Kadanoff L P and Su-Min Zhou 1993 Finite-time singularity formation in Hele-Shaw systems Phys. Rev. E 47(6) 4182-96

[17] E B Dussan V and Davis S 1974 On the motion of a fluid-fuid interface along a solid surface J. Fluid Mech. $6571-95$

[18] Greenspan H P 1978 On the motion of a small viscous droplet that wets a surface J. Fluid Mech. 84 125-43

[19] Chun Huh and Scriven L E 1971 Hydrodynamic model of steady movement of a solid /iquid/fuid contact line J. Colloid Interface Sci. 35 85-101

[20] Israelachvili J 1985 Intermolecular and surface forces (New York: Academic)

[21] Kamenomostskaya S 1973 The asymptotic behaviour of the solution of the filtration equation Israel J. Math. $1476-87$

[22] Leger L and Joanny I F 1993 Liquid spreading

[23] Lions J L 1969 Quelque méthodes de résolution des problèmes aux limites non linéaires (Paris: Dunod)

[24] Novick-Cohen A 1993 A singular minimization problem for droplet profiles Eur. J. Appl. Math. 4 399-418

[25] Peletier L A 1981 The porous media equation Applications of nonlinear analysis in the physical sciences ed H Amman (London: Pitman) pp 229-41

[26] Scheludko A 1968 Adv. Colloid Interface Sci. 1

[27] Starov V M 1983 Spreading of droplets of nonvolatile liquids over a flat solid J. Colloid Interface Sci. USSR 45

[28] Williams M B and Davis S H 1982 Nonlinear theory of film rupture J. Colloid Interface Sci. 90

[29] Zhou S 1992 Interface dynamics: bubble growth and droplet breakup in the Hele-Shaw cell PhD thesis University of Chicago 